\title{
Operator Methods and $S U(1,1)$ Symmetry in the Theory of Jacobi and of Ultraspherical Polynomials
}

\author{
Alfred Wünsche \\ Formerly: Institut für Physik, Humboldt-Universität, Nichtklassische Strahlung (Max-Planck-Group), Berlin, Germany \\ Email: alfred.wuensche@physik.hu-berlin.de
}

How to cite this paper: Wünsche, A. (2017) Operator Methods and $S U(1,1)$ Symmetry in the Theory of Jacobi and of Ultraspherical Polynomials. Advances in Pure Mathematics, 7, 213-261.

https://doi.org/10.4236/apm.2017.72012

Received: December 11, 2016

Accepted: February 13, 2017

Published: February 16, 2017

Copyright $\odot 2017$ by author and Scientific Research Publishing Inc. This work is licensed under the Creative Commons Attribution International License (CC BY 4.0).

http://creativecommons.org/licenses/by/4.0/

\section{Abstract}

Starting from general Jacobi polynomials $P_{n}^{(\alpha, \beta)}(u)$ we derive for the Ultraspherical polynomials $P_{n}^{(\alpha, \alpha)}(u)$ as their special case a set of related polynomials $G_{n}^{\alpha}(x)$ which can be extended to an orthogonal set of functions with interesting properties. It leads to an alternative definition of the Ultraspherical polynomials by a fixed integral operator in application to powers of the variable $u$ in an analogous way as it is possible for Hermite polynomials. From this follows a generating function which is apparently known only for the Legendre and Chebyshev polynomials as their special case. Furthermore, we show that the Ultraspherical polynomials form a realization of the $S U(1,1)$ Lie algebra with lowering and raising operators which we explicitly determine. By reordering of multiplication and differentiation operators we derive new operator identities for the whole set of Jacobi polynomials which may be applied to arbitrary functions and provide then function identities. In this way we derive a new "convolution identity" for Jacobi polynomials and compare it with a known convolution identity of different structure for Gegenbauer polynomials. In short form we establish the connection of Jacobi polynomials and their related orthonormalized functions to the eigensolution of the Schrödinger equation to Pöschl-Teller potentials.

\section{Keywords}

Orthogonal Polynomials, Lie Algebra $S U(1,1)$ and Lie Group $S U(1,1)$, Lowering and Raising Operators, Jacobi Polynomials, Ultraspherical Polynomials, Gegenbauer Polynomials, Chebyshev Polynomials, Legendre Polynomials, Stirling Numbers, Hypergeometric Function, Operator Identities, Vandermond's Convolution Identity, Pöschl-Teller Potentials 


\section{Introduction}

The applications of the Classical Orthogonal polynomials in physical sciences are immense. To the Classical Orthogonal polynomials belong in one-dimensional case (one variable) the Jacobi polynomials with their special case of Ultraspherical polynomials the last including the Legendre polynomials and the Chebyshev polynomials of first and second kind and on the other side the Hermite and Laguerre polynomials. The last are better classified as two-dimensional polynomials with relicts and close transformation relations to both the Hermite and the Laguerre polynomials in one-dimensional case.

One of the best older sources about Classical Orthogonal polynomials in general theory and to the Jacobi polynomials, in particular, is the monograph of Szegö [1] who also gave the modern and most general definition of this class of polynomials with the symmetries already implemented in the notation $P_{n}^{(\alpha, \beta)}(x)$ and with the best standardization. A recommendable older representation with a chapter about Jacobi polynomials which uses already its modern definition is contained in the book of Jackson [2]. A very comprehensive work with a chapter about the Classical Orthogonal polynomials is in the second volume of the monographs of Bateman and Erd'elyi [3] about Higher Transcendental Functions and also very comprehensive are the Collections of Formulae and Tables of Magnus, Oberhettinger and Soni [4] and of Gradshteyn and Ryzhik [5] and, furthermore, the collaborative work of Abramomitz and Stegun [6] with the chapter of Hochstrasser about Orthogonal polynomials. A monograph from Kusnetsov [7] has a long chapter about Orthogonal polynomials but it is not translated into English. However, the next two monographs to cite are translated into English. A very readable book with a large chapter about Orthogonal polynomials which includes many of their applications is that of Lebedev [8] and also another book in Russian of this time from Nikiforov and Uvarov [9] presents the general theoy of the Classical Orthogonal polynomials and its specialization together with applications in an appealing way. Also very readable is the monograph of Rainville [10] about special functions including the general theory of orthogonal poynomials and more detailed representations about the Jacobi polynomials and of ultraspherical polynomials or equivalently the Gegenbauer polynomials and their special cases of Legendre polynomials. In the work of Luke [11] we also find a large representative chapter about orthogonal polynomials with the general theory and with detailed consideration of Jacobi polynomials and of their special cases. A derivation of all one-dimensional Classical Orthogonal polynomials from a general definition and with proof of their completeness one finds in the monograph of Chihara [12]. A representation of the Classical Orthogonal polynomials including the Jacobi polynomials (with notation $\left.P_{n}(x ; \alpha, \beta)\right)$ and, in particular, detailed about the Chebyshev polynomials and the Legendre poynomials and also with a representation of the general theory is the monograph of Suyetin [13] (see also [14] of the same author about polynomials of two variables). A detailed representation about generating functions for polynomials and special functions and with a lot of 
formulae difficult to find at other places is the monograph of Srivastava and Manocha [15].

In more recent time, the collaborative NIST-handbook [16] of Mathematical Functions with the chapter of Koornwinder, Wong, Koekoek and Swarttouw [17] about Orthogonal polynomials is a very comprehensive work. A modern introduction to Orthogonal polynomials is contained in the monograph of Andrews, Askey and Roy [18] (see also [19]).

The representation of the Jacobi polynomials in a chapter of the monograph of Carlson [20] is a little uncommon and unusual but possibly more general as in other representations (biorthogonality with Jacobi functions) and the author generally does not write the upper indices of the Jacobi polynomials (it is difficult then to consider contiguous relations where they change in one formula) and it deals with also Laguerre polynomials under the heading of the chapter about Jacobi polynomials. A special monograph about the Chebyshev polynomials with their application in mathematical approximation theory but with only very shortly mentioning (p. 35) their superclasses of Ultraspherical polynomials, Gegenbauer polynomials and of Jacobi polynomials is that of Rivlin [21]. In short form one finds a lot of formulae for Orthogonal polynomials also in a book of Bell [22] which we cite mainly for a formula to Hermite polynomials (see footnote in Section 5). Possibly, there exist further excellent representations of the Orthogonal polynomials.

Many classes of polynomials and of Higher Transcendental Functions possess a group-theoretical background and were intensively studied in application of quantum mechanics to the theory of angular momentum that means to the irreducible representations of the three-dimensional rotation group $S O(3, \mathbb{R})$ and of spin $S U(2) \cong S O(3, \mathbb{R})$ (Spherical harmonics), in particular, by Wigner [23], Weyl [24] and Van der Waerden [25] as pioneers. The classical representation from the mathematical side is given in the monograph of Vilenkin [26]. It rests to a considerable part on the work of the Russian school of mathematicians to the representations of groups, in particular, of Gelfand, Naimark and others and of Vilenkin himself (e.g., [27] [28]). Other representations of a group-theoretical background of Orthogonal polynomials and Special functions are given, e.g., by Miller [29] [30] [31] and by Van der Jeugt [32] in the collection [33]. The monograph of Dunkl [34] is about Orthogonal polynomials of several variables (see also already cited [14]) but it treats in addition their grouptheoretical background.

The main purpose of this article is to join an approach to the Ultraspherical polynomials which leads, in particular, to an alternative definition of this class of polynomials in comparison to the Rodrigues definition with the treatment of the Ultraspherical polynomials as a realization of the (infinite-dimensional) unitary irreducible representations of the group $S U(1,1)$. We determine the lowering and raising operators in this realization of $S U(1,1)$ by the orthonormalized functions to the Ultraspherical polynomials with fixed upper index as parameter. Furthermore, we find an operator identity for the more general case of Jacobi 
polynomials which among others leads to a convolution identity for the Jacobi polynomials similarly to the Vandermond convolution formula for the binomial coefficients. We also have accumulated in the past some more or less new applications of Ultraspherical polynomials mainly connected with 2D-matrices and functions of them and of their special cases of Chebyshev polynomials which, however, we cannot include into this article. In recent time we elaborated in detail the Weyl correspondence of classical phase-space functions to quantum-mechanical operators where the Jacobi polynomials find their application in many facets and not only in their simplest special cases. A main result was shortly communicated in [35] but the material for representation became too much to be packed into present work and we intend to make this separately.

Some results and formulae in the article are formulated for the whole set of Jacobi polynomials and some only for its subset of Ultraspherical polynomials. The reason for this restriction to Ultraspherical polynomials was in these cases that we could not obtain up to now a generalization for the whole set of Jacobi polynomials which may be fairly difficult as some generating functions for both sets suggest.

\section{Basic Relations for General Jacobi Polynomials}

We give in this Section without a proof a few basic known formulae for the general Jacobi polynomials $P_{n}^{(\alpha, \beta)}(u)$. The integers $n,(n=0,1,2, \cdots)$ denote the degree of the polynomials in the variable $u$ which is a real or complex variable and $\alpha$ and $\beta$ are two parameters which usually take on real values. A lot of formulae for them can be found in [1] and in short form in [3] [5] [6] [17]. The Ultraspherical polynomials $P_{n}^{(\alpha, \alpha)}(u)$ are the special case $\alpha=\beta$ of the Jacobi polynomials $P_{n}^{(\alpha, \beta)}(u)$. The class of Ultraspherical is equivalent to the class of Gegenbauer polynomials $C_{n}^{v}(u)$ (see Section 3).

The Jacobi polynomials were defined in the now generally accepted notation $P_{n}^{(\alpha, \beta)}(u)$ by Szegö [1] in the Rodrigues-like form for Classical Orthogonal polynomials (e.g., [3]) as follows

$$
P_{n}^{(\alpha, \beta)}(u)=\frac{(-1)^{n}}{2^{n} n !} \frac{1}{(1-u)^{\alpha}(1+u)^{\beta}} \frac{\partial^{n}}{\partial u^{n}}(1-u)^{n+\alpha}(1+u)^{n+\beta},
$$

from which by applying the Leibniz rule for the multiple differentiation of a product of functions follows immediately the explicit representation

$$
P_{n}^{(\alpha, \beta)}(u)=\frac{1}{2^{n}} \sum_{k=0}^{n} \frac{(n+\alpha) !(n+\beta) !}{k !(n-k) !(n+\alpha-k) !(k+\beta) !}(u-1)^{n-k}(u+1)^{k} .
$$

Using the commutation relation of the operator $\frac{\partial}{\partial u}$ with the operator

$(1-u)^{\alpha}(1+u)^{\beta} \equiv w^{(\alpha, \beta)}(u)$ which is the weight function of the Jacobi polynomials we find from (2.1) the following useful modification of the definition

$$
P_{n}^{(\alpha, \beta)}(u)=\frac{1}{2^{n} n !}\left(\frac{\partial}{\partial u}+\frac{\alpha}{u-1}+\frac{\beta}{u+1}\right)^{n}\left(u^{2}-1\right)^{n},
$$


and if we bring the function $\left(u^{2}-1\right)^{n}$ in front of the differentiation operator

$$
P_{n}^{(\alpha, \beta)}(u)=\frac{1}{2^{n} n !}\left(u^{2}-1\right)^{n}\left(\frac{\partial}{\partial u}+\frac{n+\alpha}{u-1}+\frac{n+\beta}{u+1}\right)^{n} 1 .
$$

The standardization of the Jacobi polynomials is

$$
P_{0}^{(\alpha, \beta)}(u)=1, \quad P_{n}^{(\alpha, \beta)}(1)=\frac{(n+\alpha) !}{n ! \alpha !}, P_{n}^{(\alpha, \beta)}(-1)=(-1)^{n} \frac{(n+\beta) !}{n ! \beta !} .
$$

In [1] are also given relations to older definitions by Jacobi, Jordan, Stieltjes, Courant and Hilbert and Fejér. The Jacobi polynomials belong to the Classical Orthogonal Polynomials which obey a second-order differential equation. The integers $n,(n=0,1,2, \cdots)$, are the degree of the polynomials but it is not possible to find here a simple explicit expansion in powers of the variable $u$ because for their determination we need the values $P_{n}^{(\alpha, \beta)}(0)$ for which more compact relations others than than that specialized from the general sum expression (2.2) or equivalent ones (see (2.8)) do, in general, not exist.

Using two free parameters $\gamma$ and $\delta$ one may insert an intermediate step in the transition from definition (2.1) to representation (2.3) according to

$$
\begin{aligned}
& P_{n}^{(\alpha, \beta)}(u) \\
= & \frac{(-1)^{n}}{2^{n} n !} \frac{1}{(1-u)^{\alpha}(1+u)^{\beta}} \frac{\partial^{n}}{\partial u^{n}}(1-u)^{n+\alpha}(1+u)^{n+\beta} \\
= & \frac{(-1)^{n}}{2^{n} n !} \frac{1}{(1-u)^{\gamma}(1+u)^{\delta}}\left(\frac{\partial}{\partial u}+\frac{\alpha-\gamma}{u-1}+\frac{\beta-\delta}{u+1}\right)^{n}(1-u)^{n+\gamma}(1+u)^{n+\delta} .
\end{aligned}
$$

This relation becomes important in Section 12 when we derive a convolution identity for Jacobi polynomials.

The Jacobi polynomials $P_{n}^{(\alpha, \beta)}(u)$ are special cases of the Hypergeometric function ${ }_{2} F_{1}(a, b ; c ; z)$ as follows

$$
\begin{aligned}
& P_{n}^{(\alpha, \beta)}(u) \\
= & \frac{(n+\beta) !}{n ! \beta !}\left(\frac{u-1}{2}\right)_{2}^{n} F_{1}\left(-n,-n-\alpha ; 1+\beta ; \frac{u+1}{u-1}\right) \\
= & \left(\frac{u-1}{2}\right)^{n} \sum_{k=0}^{n} \frac{(n+\alpha) !(n+\beta) !}{k !(n-k) !(n+\alpha-k) !(\beta+k) !}\left(\frac{u+1}{u-1}\right)^{k},
\end{aligned}
$$

where starting from the basic definition of the Hypergeometric series of ${ }_{2} F_{1}(a, b ; c ; z)$ one has to apply the transformation of the factorials $\frac{(k+a-1) !}{(a-1) !}=\frac{(-1)^{k}(-a) !}{(-a-k) !}$ and correspondingly for $b$ instead of $a$. By a known general transformation relation of the Hypergeometric function ${ }_{2} F_{1}(a, b ; c ; z)$ where the variable $z$ transforms into the variable $\frac{z}{z-1}$ (e.g., [36], chap. (2.1.4), Equation (22)) and which goes back to Gauss the Jacobi polynomials can be explicitly represented also by 


$$
\begin{aligned}
P_{n}^{(\alpha, \beta)}(u) & =(-1)^{n} \frac{(n+\beta) !}{n ! \beta !}{ }_{2} F_{1}\left(-n, n+\alpha+\beta+1 ; 1+\beta ; \frac{u+1}{2}\right) \\
& =\frac{(-1)^{n}(n+\beta) !}{(n+\alpha+\beta) !} \sum_{j=0}^{n} \frac{(n+\alpha+\beta+j) !}{j !(n-j) !(j+\beta) !}\left(-\frac{1+u}{2}\right)^{j},
\end{aligned}
$$

or equivalently by substitution $j=n-k$ of the summation index

$$
P_{n}^{(\alpha, \beta)}(u)=\frac{(n+\beta) !}{2^{n}(n+\alpha+\beta) !} \sum_{k=0}^{n} \frac{(-1)^{k} 2^{k}(2 n+\alpha+\beta-k) !}{k !(n-k) !(n+\beta-k) !}(1+u)^{n-k} .
$$

The transition from (2.7) to (2.8) can also be made directly on the level of the polynomials that is demonstrated in Appendix A.

From (2.1) follows the symmetry property

$$
P_{n}^{(\alpha, \beta)}(u)=(-1)^{n} P_{n}^{(\beta, \alpha)}(-u) .
$$

In connection of (2.7) and (2.8) with this symmetry one may write down 4 equivalent definitions of the Jacobi polynomials by the Hypergeometric function ${ }_{2} F_{1}(a, b ; c ; z)$ which all are given in [3] (Chap. 10.8, Equation (16)).

By differentiation of $P_{n}^{(\alpha, \beta)}(u)$ with respect to variable $u$ one finds (most easily) using (2.8)

$$
\begin{aligned}
\frac{\partial}{\partial u} P_{n}^{(\alpha, \beta)}(u) & =\frac{n+\alpha+\beta+1}{2} P_{n-1}^{(\alpha+1, \beta+1)}(u), \\
\frac{\partial^{k}}{\partial u^{k}} P_{n}^{(\alpha, \beta)}(u) & =\frac{(n+\alpha+\beta+k) !}{2^{k}(n+\alpha+\beta) !} P_{n-k}^{(\alpha+k, \beta+k)}(u), \\
\frac{\partial^{n}}{\partial u^{n}} P_{n}^{(\alpha, \beta)}(u) & =\frac{(2 n+\alpha+\beta) !}{2^{n}(n+\alpha+\beta) !} P_{=1}^{(\alpha+n, \beta+n)}(u)=\frac{(2 n+\alpha+\beta) !}{2^{n}(n+\alpha+\beta) !}
\end{aligned}
$$

From this follows the expansion of $P_{n}^{(\alpha, \beta)}(u)$ in powers of $u$

$$
\begin{aligned}
P_{n}^{(\alpha, \beta)}(u) & =\sum_{k=0}^{n} \frac{1}{k !}\left(\frac{\partial^{k}}{\partial x^{k}} P_{n}^{(\alpha, \beta)}(x)\right)_{x=0} u^{k} \\
& =\sum_{k=0}^{n} \frac{(n+\alpha+\beta+k) !}{2^{k} k !(n+\alpha+\beta) !} P_{n-k}^{(\alpha+k, \beta+k)}(0) u^{k} .
\end{aligned}
$$

Apart from the special case $\alpha=\beta$ which we consider beginning with Section 3 and a few other special cases which we do not discuss here it is not possible to give for the Jacobi polynomials $P_{n}^{(\alpha, \beta)}(0)$ of argument zero simple formulae of multiplicative type others than the sum formulae which follow from the basic explicit relations for $P_{n}^{(\alpha, \beta)}(u)$ by specialization.

We abbreviate the weight function of the Jacobi polynomials $P_{n}^{(\alpha, \beta)}(u)$ in the interval $[-1,+1]$ by $w^{(\alpha, \beta)}(u)$ as follows

$$
w^{(\alpha, \beta)}(u) \equiv(1-u)^{\alpha}(1+u)^{\beta} .
$$

Using the recurrence relation

$$
\left(1-u^{2}\right) \frac{\partial}{\partial u} P_{n}^{(\alpha, \beta)}(u)=(\alpha-\beta+(\alpha+\beta) u) P_{n}^{(\alpha, \beta)}(u)-2(n+1) P_{n+1}^{(\alpha-1, \beta-1)}(u),
$$


which can be checked by the given explicit representations for the Jacobi polynomials one may derive the following well-known second-order differential equation for the Jacobi polynomials (e.g, [1] [3])

$$
\left\{\frac{\partial}{\partial u}\left(1-u^{2}\right) \frac{\partial}{\partial u}-(\alpha-\beta+(\alpha+\beta) u) \frac{\partial}{\partial u}+n(n+\alpha+\beta+1)\right\} P_{n}^{(\alpha, \beta)}(u)=0 .
$$

We introduce now the functions $p_{n}^{(\alpha, \beta)}(u)$ by

$$
p_{n}^{(\alpha, \beta)}(u) \equiv \sqrt{\frac{2 n+\alpha+\beta+1}{2^{\alpha+\beta+1}} \frac{n !(n+\alpha+\beta) !}{(n+\alpha) !(n+\beta) !}} \sqrt{w^{(\alpha, \beta)}(u)} P_{n}^{(\alpha, \beta)}(u) .
$$

They are orthonormalized in the interval $[-1,+1]$ according to

$$
\int_{-1}^{+1} \mathrm{~d} u p_{m}^{(\alpha, \beta)}(u) p_{n}^{(\alpha, \beta)}(u)=\delta_{m, n}, \quad(\alpha>-1, \beta>-1) .
$$

Connected with these orthonormality relations are the completeness relations in the interval $[-1,+1]$

$$
\sum_{n=0}^{\infty} p_{n}^{(\alpha, \beta)}(u) p_{n}^{(\alpha, \beta)}(v)=\delta(u-v), \quad(\alpha>-1, \beta>-1) .
$$

In both relations (2.17) and (2.18) the parameters $(\alpha, \beta)$ apart from the restrictions $\alpha>-1, \beta>-1$ can be chosen within wide borders.

Using transformation relations for the operators in the differential equation one may transform it, for example, to the following form

$$
\begin{aligned}
& \left\{\frac{1}{4}\left(\sqrt{1-u^{2}} \frac{\partial}{\partial u}+\frac{\partial}{\partial u} \sqrt{1-u^{2}}\right)^{2}-\frac{1}{2}\left(\frac{\alpha^{2}-\frac{1}{4}}{1-u}+\frac{\beta^{2}-\frac{1}{4}}{1+u}\right)+\left(n+\frac{\alpha+\beta+1}{2}\right)^{2}\right\} p_{n}^{(\alpha, \beta)}(u) \\
& =0 .
\end{aligned}
$$

with a self-adjoint operator in front of $p_{n}^{(\alpha, \beta)}(u)$. A slightly modified in form but fully equivalent representation of this differential equation is derived by Szegö [1] (Chap. IV, Equations (4.24.1) and (4.24.2)).

We mention here shortly that Equation (2.19) is equivalent to the Schrödinger equation for the Pöschl-Teller potentials (e.g., [37] [38]). For this purpose we make in (2.19) the substitution

$$
\begin{aligned}
& u=\cos (x), \sqrt{1-u^{2}}=\sin (x), \frac{\partial}{\partial u}=-\frac{1}{\sin (x)} \frac{\partial}{\partial x}, \\
& \mathrm{~d} u=-\sin (x) \mathrm{d} x, 0 \leq x \leq \pi .
\end{aligned}
$$

which transforms the interval $[-1,+1]$ for $u$ into the interval $[0, \pi]$ for $x$. From the orthonormalization of the functions $p_{n}^{(\alpha, \beta)}(u)$ follows that now the states $\psi_{n}^{(\alpha, \beta)}(x)$ defined by ${ }^{1}$

${ }^{1}$ We mention that in [37] and also in [38] the eigensolutions $\psi_{n}(x)$ are represented by the Hypergeometric function ${ }_{2} F_{1}(a, b ; c ; z)$ without a hint to the possible representation by the Jacobi polynomials. 


$$
\begin{aligned}
& \psi_{n}^{(\alpha, \beta)}(x) \\
& \equiv \sqrt{(2 n+\alpha+\beta+1) \frac{n !(n+\alpha+\beta) !}{(n+\alpha) !(n+\beta) !}} \sin ^{\alpha+\frac{1}{2}}\left(\frac{x}{2}\right) \cos ^{\beta+\frac{1}{2}}\left(\frac{x}{2}\right) P_{n}^{(\alpha, \beta)}(\cos (x)) \\
& =\sqrt{\sin (x)} p_{n}^{(\alpha, \beta)}(\cos (x)), \Rightarrow \psi_{n}^{(\alpha, \beta)}(0)=\psi_{n}^{(\alpha, \beta)}(\pi)=0,\left(\alpha>-\frac{1}{2}, \beta>-\frac{1}{2}\right),
\end{aligned}
$$

are orthonormalized in the interval $0 \leq x \leq \pi$ according to

$$
\int_{0}^{\pi} \mathrm{d} x \psi_{m}^{(\alpha, \beta)}(x) \psi_{n}^{(\alpha, \beta)}(x)=\delta_{m, n} .
$$

The first derivative of $\psi_{n}^{(\alpha, \beta)}(x)$ is

$$
\begin{aligned}
& \frac{\partial}{\partial x} \psi_{n}^{(\alpha, \beta)}(x) \\
& \equiv \sqrt{(2 n+\alpha+\beta+1) \frac{n !(n+\alpha+\beta) !}{(n+\alpha) !(n+\beta) !}} \sin ^{\alpha+\frac{3}{2}}\left(\frac{x}{2}\right) \cos ^{\beta+\frac{3}{2}}\left(\frac{x}{2}\right) \\
& \cdot\left\{\frac{1}{2}\left(\frac{\alpha+\frac{1}{2}}{\sin ^{2}\left(\frac{x}{2}\right)}-\frac{\beta+\frac{1}{2}}{\cos ^{2}\left(\frac{x}{2}\right)}\right) P_{n}^{(\alpha, \beta)}(\cos (x))-(n+\alpha+\beta+1) P_{n-1}^{(\alpha+1, \beta+1)}(\cos (x))\right\} .
\end{aligned}
$$

From (2.19) we find a differential equation for $\psi_{n}^{(\alpha, \beta)}(x)$ which possesses the form of a stationary Schrödinger equation for a Hamilton operator $H$ with a potential function $U(x)$ in form of an asymmetric $\left(\alpha \neq \beta ; \alpha^{2}>\beta^{2}>\frac{1}{4}\right)$ hole with infinitely high walls at $x=0$ and $x=\pi$ (or abyss at $x=\pi$ for $\alpha^{2}<\beta^{2}$ ) to energy eigenvalues $E_{n}$ according to (we set $\hbar=1$ and masses also equal to 1 )

$$
H \psi_{n}^{(\alpha, \beta)}(x) \equiv\left(-\frac{1}{2} \frac{\partial^{2}}{\partial x^{2}}+U(x)\right) \psi_{n}^{(\alpha, \beta)}(x)=E_{n} \psi_{n}^{(\alpha, \beta)}(x),
$$

which explicitly possesses the form

$$
\begin{aligned}
& \left\{-\frac{1}{2} \frac{\partial^{2}}{\partial x^{2}}+\frac{\alpha^{2}+\beta^{2}-\frac{1}{2}+\left(\alpha^{2}-\beta^{2}\right) \cos (x)}{4 \sin ^{2}(x)}\right\} \psi_{n}^{(\alpha, \beta)}(x) \\
& =\left\{-\frac{1}{2} \frac{\partial^{2}}{\partial x^{2}}+\frac{1}{8}\left(\frac{\alpha^{2}-\frac{1}{4}}{\sin ^{2}\left(\frac{x}{2}\right)}+\frac{\beta^{2}-\frac{1}{4}}{\cos ^{2}\left(\frac{x}{2}\right)}\right)\right\} \psi_{n}^{(\alpha, \beta)}(x)=\frac{1}{2}\left(n+\frac{\alpha+\beta+1}{2}\right)^{2} \psi_{n}^{(\alpha, \beta)}(x) .
\end{aligned}
$$

The upper indices $\alpha>-1$ and $\beta>-1$ are two independent parameters and their squares $\alpha^{2}$ and $\beta^{2}$ determine the potential function $U(x)$ which are the Pöschl-Teller potentials and the energy eigenvalues depend quadratically on $n$. The walls at $x=0$ and $x=\pi$ are supposed to be impenetrable according 
to boundary conditions in (2.21) and in other case the results have to be modified in dependence on the boundary conditions. Furthermore, there are peculiarities with the infinite walls at the boundaries which become infinite abysses at $x=0$ for $\alpha^{2}<\frac{1}{4}$ and at $x=\pi$ for $\beta^{2}<\frac{1}{4}$. To study all this in systematic way and, for example, also the possible continuation of the potentials for all real $x$ in periodic way is not our intention here.

The completeness relation (2.18) allows to make expansions of functions $f(u)$ in the interval $[-1,1]$ in series of the Jacobi polynomials $P_{n}^{(\alpha, \beta)}(u)$ according to

$$
\begin{aligned}
f(u) & =\sum_{n=0}^{\infty} c_{n}^{(\alpha, \beta)} P_{n}^{(\alpha, \beta)}(u), \\
c_{n}^{(\alpha, \beta)} & =\frac{2 n+\alpha+\beta+1}{2^{\alpha+\beta+1}} \frac{n !(n+\alpha+\beta) !}{(n+\alpha) !(n+\beta) !} \int_{-1}^{+1} \mathrm{~d} v w^{(\alpha, \beta)}(v) P_{n}^{(\alpha, \beta)}(v) f(v),
\end{aligned}
$$

or, more generally, in series of the set of functions $\left(w^{(\alpha, \beta)}(u)\right)^{\lambda} P_{n}^{(\alpha, \beta)}(u)$ with additional widely arbitrary parameter $\lambda$ according to

$$
\begin{aligned}
& f(u)=\sum_{n=0}^{\infty} d_{n}^{(\lambda ; \alpha, \beta)}\left(w^{(\alpha, \beta)}(u)\right)^{\lambda} P_{n}^{(\alpha, \beta)}(u), \\
& d_{n}^{(\lambda ; \alpha, \beta)}=\frac{2 n+\alpha+\beta+1}{2^{\alpha+\beta+1}} \frac{n !(n+\alpha+\beta) !}{(n+\alpha) !(n+\beta) !} \int_{-1}^{+1} \mathrm{~d} v\left(w^{(\alpha, \beta)}(v)\right)^{1-\lambda} P_{n}^{(\alpha, \beta)}(v) f(v),
\end{aligned}
$$

if the function $f(u)$ is well-behaved enough to guarantee the existence of the involved integrals.

As example for the inversion of the expression of the Jacobi polynomials $P_{n}^{(\alpha, \beta)}(u)$ in powers of $\frac{1+u}{2}$ we found (see also [10], p. 62, Equation (2))

$$
\begin{aligned}
& \left(\frac{1+u}{2}\right)^{n} \\
& =\sum_{k=0}^{n}(2 n+\alpha+\beta+1-2 k) \frac{(n+\beta) !}{k !(n+\beta-k) !} \frac{n !(n+\alpha+\beta-k) !}{(2 n+\alpha+\beta+1-k) !} P_{n-k}^{(\alpha, \beta)}(u),
\end{aligned}
$$

where $(\alpha, \beta)$ are parameters which can be chosen arbitrarily. The expansion of $u^{n}$ in a sum over Jacobi polynomials is

$u^{n}$

$=2^{n} n ! \sum_{k=0}^{n} \frac{(2 n+\alpha+\beta+1-2 k)(n+\alpha+\beta-k) !}{(2 n+\alpha+\beta+1-k) !} P_{k}^{(-n-\alpha-1,-n-\beta-1)}(0) P_{n-k}^{(\alpha, \beta)}(u)$.

Apparently, the last relation is new.

From a general transformation relation of the Hypergeometric function ${ }_{2} F_{1}(a, b ; c ; z)$ where the variable $z$ is transformed to the variable

$z \rightarrow z^{\prime}=\frac{z}{z-1}$ ([5], first two of Equations (9.131), [36], Equation 2.1. (23) and (24)) follows via the connection with the Jacobi polynomials the following general transformation with change of the variable $u$ of the Jacobi polynomials to 
$u \rightarrow u^{\prime}=\frac{3+u}{1-u}$ and with symmetry (2.10) (Szegö [1])

$$
P_{n}^{(\alpha, \beta)}(u)=\left(\frac{1+u}{2}\right)^{n} P_{n}^{(\alpha,-2 n-\alpha-\beta-1)}\left(\frac{3-u}{1+u}\right)
$$

with fixed points $u=+1,-3$. The argument $u$ is here transformed by a certain fractional linear transformation (Möbius transformation) to the new argument.

There exists yet another transformation relation for the Jacobi polynomials $P_{n}^{(\alpha, \beta)}(u)$ which is only possible if one of the upper indices $\alpha$ or $\beta$ is an integer $k \geq-n$ and which we write in the form

$$
\left(\frac{1+u}{2}\right)^{k} P_{n}^{(\alpha, k)}(u)=\frac{(n+\alpha) !(n+k) !}{n !(n+\alpha+k) !} P_{n+k}^{(\alpha,-k)}(u) .
$$

Its origin comes also from one of the general transformation relations for the Hypergeometric function ${ }_{2} F_{1}(a, b ; c ; z)$ if in addition to $a$ or $b$ equal to negative integers $-n$ (polynomial case) the third parameter $c$ is equal to an integer $k \geq-n$ (see, e.g., [5], third of Equations 9.131, [36], Equation 2.1.(23)) In other case the relation is also true but leads outside from the Jacobi polynomials to the corresponding Hypergeometric function since the lower index $n+k$ is then no more an integer.

The basic recurrence relation for the Jacobi polynomials with fixed upper indices possesses the form (e.g., [1] [3] and others)

$$
\begin{aligned}
& 2(n+1)(n+\alpha+\beta+1)(2 n+\alpha+\beta) P_{n+1}^{(\alpha, \beta)}(u) \\
= & (2 n+\alpha+\beta+1)\left((2 n+\alpha+\beta)(2 n+\alpha+\beta+2) u+\alpha^{2}-\beta^{2}\right) P_{n}^{(\alpha, \beta)}(u) \\
& -2(n+\alpha)(n+\beta)(2 n+\alpha+\beta+2) P_{n-1}^{(\alpha, \beta)}(u) .
\end{aligned}
$$

The relation for lowering of the lower index $n$ of the Jacobi polynomials $P_{n}^{(\alpha, \beta)}(u)$ preserving the two upper indices $(\alpha, \beta)$ as parameter is

$$
\begin{aligned}
& \left\{(2 n+\alpha+\beta)\left(n u+\left(1-u^{2}\right) \frac{\partial}{\partial u}\right)-n(\alpha-\beta)\right\} P_{n}^{(\alpha, \beta)}(u) \\
& =2(n+\alpha)(n+\beta) P_{n-1}^{(\alpha, \beta)}(u),
\end{aligned}
$$

and the corresponding relation for raising the index

$$
\begin{aligned}
& \left\{(2 n+\alpha+\beta+2)\left((n+\alpha+\beta+1) u-\left(1-u^{2}\right) \frac{\partial}{\partial u}\right)+(n+\alpha+\beta+1)(\alpha-\beta)\right\} \\
& \cdot P_{n}^{(\alpha, \beta)}(u) \\
& =2(n+1)(n+\alpha+\beta+1) P_{n+1}^{(\alpha, \beta)}(u) .
\end{aligned}
$$

There is a large number of contiguous relations where in addition to the lower index $n$ or alone also one or both upper indices $(\alpha, \beta)$ are changed by steps of 1 . We are mainly concerned in this paper with relations where the upper indices $(\alpha, \beta)$ are fixed parameters and therefore we do not give them.

\section{Basic Relations for Ultraspherical Polynomials}

As already said the special case $\alpha=\beta$ of the Jacobi polynomials $P_{n}^{(\alpha, \beta)}(u)$ is 
called the Ultraspherical polynomials $P_{n}^{(\alpha, \alpha)}(u)$. The Ultraspherical polynomials are in a one-to-one correspondence to the Gegenbauer polynomials with the standard notation $C_{n}^{v}(u)$ and this relation is ${ }^{2}$

$$
\begin{aligned}
& P_{n}^{(\alpha, \alpha)}(u)=\frac{(n+\alpha) !(2 \alpha) !}{\alpha !(n+2 \alpha) !} C_{n}^{\alpha+\frac{1}{2}}(u), \\
& C_{n}^{v}(u)=\frac{(n+2 v-1) !\left(v-\frac{1}{2}\right) !}{(2 v-1) !\left(n+v-\frac{1}{2}\right) !} P_{n}^{\left(v-\frac{1}{2}, v-\frac{1}{2}\right)}(u) .
\end{aligned}
$$

In the following we do not translate every formula for the Ultraspherical polynomials into a representation by the Gegenbauer polynomials. Furthermore, some of the Ultraspherical polynomials with a low fixed upper index due to their importance in applications got special names and notations. These are the Chebyshev polynomials of first kind (upper index $\alpha=-\frac{1}{2}$ with notation $T_{n}(u)$ and of second kind with upper index $\alpha=\frac{1}{2}$ with notation $\left.U_{n}(u)\right)$ and the Legendre polynomials (upper index $\alpha=0$ ) with notation $P_{n}(u)$ as follows

$$
\begin{aligned}
& T_{n}(u)=\frac{2^{2 n} n !^{2}}{(2 n) !} P_{n}^{\left(-\frac{1}{2},-\frac{1}{2}\right)}(u)=\frac{n !\left(-\frac{1}{2}\right) !}{\left(n-\frac{1}{2}\right) !} P_{n}^{\left(-\frac{1}{2},-\frac{1}{2}\right)}(u)=\frac{n}{2} \lim _{\varepsilon \rightarrow 0} \frac{1}{\varepsilon} C_{n}^{\varepsilon}(u), \\
& \left(C_{n}^{0}(u)=\delta_{n, 0}\right), \\
& P_{n}(u)=P_{n}^{(0,0)}(u)=C_{n}^{\frac{1}{2}}(u), \\
& U_{n}(u)=\frac{2^{2 n} n !(n+1) !}{(2 n+1) !} P_{n}^{\left(\frac{1}{2}, \frac{1}{2}\right)}(u)=(n+1) \frac{n !\left(\frac{1}{2}\right) !}{\left(n+\frac{1}{2}\right) !} P_{n}^{\left(\frac{1}{2}, \frac{1}{2}\right)}(u)=C_{n}^{1}(u) .
\end{aligned}
$$

The Gegenbauer polynomial $C_{n}^{v}(u)$ for upper index $v=0$ are vanishing for $n \neq 0$ and are badly appropriate for the definition of the Chebyshev polynomials of first kind $T_{n}(u)$ but their definition from the Ultraspherical polynomials is simple and unique. We derive now some of the basic relations for Ultraspherical polynomials $P_{n}^{(\alpha, \alpha)}(u)$ from the corresponding relations for Jacobi polynomials.

From (2.1) follows in case of $\alpha=\beta$ as definition of the kind of the Rodrigues-type

$$
\begin{aligned}
P_{n}^{(\alpha, \alpha)}(u) & =\frac{(-1)^{n}}{2^{n} n !} \frac{1}{\left(1-u^{2}\right)^{\alpha}} \frac{\partial^{n}}{\partial u^{n}}\left(1-u^{2}\right)^{n+\alpha} \\
& =\frac{(-1)^{n}}{2^{n} n !}\left(\frac{\partial}{\partial u}-\frac{2 \alpha u}{1-u^{2}}\right)^{n}\left(1-u^{2}\right)^{n} .
\end{aligned}
$$

${ }^{2}$ In this and many other formulae in literature it is often used the Pochhammer symbol $(x)_{n} \equiv \frac{(n+x-1) !}{(x-1) !} \equiv \frac{\Gamma(n+x)}{\Gamma(x)}$ as abbreviation for the so-called "shifted" factorials or Gamma function. 
From (2.10) follows for the symmetry of the Ultraspherical polynomials

$$
\begin{aligned}
& P_{n}^{(\alpha, \alpha)}(u)=(-1)^{n} P_{n}^{(\alpha, \alpha)}(-u), \Rightarrow P_{2 m}^{(\alpha, \alpha)}(u)=P_{2 m}^{(\alpha, \alpha)}(-u), \\
& P_{2 m+1}^{(\alpha, \alpha)}(u)=-P_{2 m+1}^{(\alpha, \alpha)}(-u), \Rightarrow P_{2 m+1}^{(\alpha, \alpha)}(0)=0 .
\end{aligned}
$$

This means that $P_{2 m}^{(\alpha, \alpha)}(u)$ and $\frac{1}{u} P_{2 m+1}^{(\alpha, \alpha)}(u)$ with $m=0,1,2, \cdots$ are even polynomials of $u$ which therefore depend only on $u^{2}$. This also means that $P_{n}^{(\alpha, \alpha)}(0)$ vanishes for odd indices $n=2 m+1$.

Concerning the transformation relations following from the transformation relations of the Hypergeometric function ${ }_{2} F_{1}(a, b ; c ; z)$ the case $\alpha=\beta$ possesses a specifics with no correspondence in the general Jacobi polynomials $P^{(\alpha, \beta)}(u)$ with $\alpha \neq \beta$. This is one of the cases where the application of the so-called quadratic transformation relations of Gauss and Kummer is possible. Specifically, this is the transformation relation given in [36] (2.1.5, Equation (28)) which transforms the Hypergeometric equation in the sum of two terms containing the Hypergeometric function on the right-hand side from which in special case of the Ultraspherical polynomials one term vanishes for even degree $n=2 m$ and the other term for odd degree $n=2 m+1$ and one hase the two separate relations $[1]$

$$
\begin{aligned}
& P_{2 m}^{(\alpha, \alpha)}(u)=\frac{(2 m+\alpha) ! m !}{(m+\alpha) !(2 m) !} P_{m}^{\left(\alpha,-\frac{1}{2}\right)}\left(2 u^{2}-1\right), \\
& P_{2 m+1}^{(\alpha, \alpha)}(u)=\frac{(2 m+1+\alpha) ! m !}{(m+\alpha) !(2 m+1) !} u P_{m}^{\left(\alpha, \frac{1}{2}\right)}\left(2 u^{2}-1\right),
\end{aligned}
$$

where the argument $u$ is transformed in a quadratic function of $u$ as new argument. This shows also that the Jacobi polynomials with unequal upper indices $\alpha \neq \beta=\mp \frac{1}{2}$ are also Ultraspherical polynomials. We mention yet in this connection that due to the transformation relation (2.30) which changes one of the upper indices and which for $\alpha=\beta$ takes on the form

$$
P_{n}^{(\alpha, \alpha)}(u)=\left(\frac{1+u}{2}\right)^{n} P_{n}^{(\alpha,-2 n-2 \alpha-1)}\left(\frac{3-u}{1+u}\right),
$$

the upper indices must not necessarily be equal for representations of Ultraspherical polynomials.

The weight function for Ultraspherical polynomials is

$$
w^{(\alpha, \alpha)}(u)=\left(1-u^{2}\right)^{\alpha} .
$$

From (2.15) we obtain the differential equation for Ultraspherical polynomials

$$
\left\{\frac{\partial}{\partial u}\left(1-u^{2}\right) \frac{\partial}{\partial u}-2 \alpha u \frac{\partial}{\partial u}+n(n+2 \alpha+1)\right\} P_{n}^{(\alpha, \alpha)}(u)=0,
$$

One of the forms of the differential equation for Ultraspherical polynomials multiplied with the square root of the weight function is 


$$
\left\{\frac{\partial}{\partial u}\left(1-u^{2}\right) \frac{\partial}{\partial u}-\frac{(\alpha u)^{2}}{1-u^{2}}+\alpha+n(n+2 \alpha+1)\right\} \sqrt{w^{(\alpha, \alpha)}(u)} P_{n}^{(\alpha, \alpha)}(u)=0 .
$$

The orthonormalized functions $p_{n}^{(\alpha, \alpha)}(u)$ defined by (see (2.16))

$$
p_{n}^{(\alpha, \alpha)}(u) \equiv \sqrt{\frac{2 n+2 \alpha+1}{2^{2 \alpha+1}} \frac{n !(n+2 \alpha) !}{(n+\alpha) !^{2}}} \sqrt{w^{(\alpha, \alpha)}(u)} P_{n}^{(\alpha, \alpha)}(u)
$$

satisfy the same differential equation as in (3.9) that means

$$
\left\{\frac{\partial}{\partial u}\left(1-u^{2}\right) \frac{\partial}{\partial u}-\frac{(\alpha u)^{2}}{1-u^{2}}+\alpha+n(n+2 \alpha+1)\right\} p_{n}^{(\alpha, \alpha)}(u)=0 .
$$

The orthonormality relation for the Ultraspherical polynomials possesses the form

$$
\int_{-1}^{+1} \mathrm{~d} u p_{m}^{(\alpha, \alpha)}(u) p_{n}^{(\alpha, \alpha)}(u)=\delta_{m, n},
$$

and the completeness relation for the interval $[-1,+1]$ according to $(2.18)$ is

$$
\sum_{n=0}^{\infty} p_{n}^{(\alpha, \alpha)}(u) p_{n}^{(\alpha, \alpha)}(v)=\delta(u-v)
$$

The operation of lowering the degree of the Ultraspherical polynomials $P_{n}^{(\alpha, \alpha)}(u)$ specialized from $(2.33)$ is

$$
\left(n u+\left(1-u^{2}\right) \frac{\partial}{\partial u}\right) P_{n}^{(\alpha, \alpha)}(u)=(n+\alpha) P_{n-1}^{(\alpha, \alpha)}(u),
$$

and the corresponding operation of raising the degree specialized from (2.34)

$$
\begin{aligned}
& (n+\alpha+1)\left((n+2 \alpha+1) u-\left(1-u^{2}\right) \frac{\partial}{\partial u}\right) P_{n}^{(\alpha, \alpha)}(u) \\
& =(n+1)(n+2 \alpha+1) P_{n+1}^{(\alpha, \alpha)}(u) .
\end{aligned}
$$

They form the starting point in Section 9 for the explicit determination of the connection of the Ultraspherical polynomials to the $S U(1,1)$ group.

\section{Explicit Expressions for the Ultraspherical Polynomials}

We now derive and compile explicit sum representations for the Ultraspherical polynomials. Using the symmetry (3.3) we find from (2.9)

$$
\begin{aligned}
& P_{n}^{(\alpha, \alpha)}(u) \\
& =\frac{(n+\alpha) !}{2^{n}(n+2 \alpha) !} \sum_{k=0}^{n} \frac{(-2)^{k}(2 n+2 \alpha-k) !}{k !(n-k) !(n+\alpha-k) !} \frac{1}{2}\left((u+1)^{n-k}+(-1)^{k}(u-1)^{n-k}\right) \\
& =\frac{(n+\alpha) !}{2^{n}(n+2 \alpha) !} \sum_{k=0}^{n} \frac{(-2)^{k}(2 n+2 \alpha-k) !}{k !(n-k) !(n+\alpha-k) !} \sum_{l=0}^{n-k} \frac{(n-k) !}{l !(n-k-l) !} u^{n-k-l} \frac{1}{2}\left(1+(-1)^{k+l}\right) .
\end{aligned}
$$

The factor $1+(-1)^{k+l}$ is only unequal to zero if $k+l$ is an even integer $2 j$

$$
\frac{1}{2}\left(1+(-1)^{k+l}\right)=\left\{\begin{array}{lc}
1, & (k+l=2 j) \\
0, & (k+l=2 j+1)
\end{array} .\right.
$$


Therefore after changing the order of summations we find

$$
P_{n}^{(\alpha, \alpha)}(u)=\frac{(n+\alpha) !}{2^{n}(n+2 \alpha) !} \sum_{j=0}^{\left[\frac{n}{2}\right.}\left(\sum_{k=0}^{2 j} \frac{(-2)^{k}(2 n+2 \alpha-k) !}{k !(n+\alpha-k) !(2 j-k) !}\right) \frac{u^{n-2 j}}{(n-2 j) !},
$$

that after evaluation of the inner sum leads to the known explicit representation

$$
P_{n}^{(\alpha, \alpha)}(u)=\frac{(n+\alpha) !}{2^{n}(n+2 \alpha) !} \sum_{j=0}^{\left[\frac{n}{2}\right]} \frac{(-1)^{j}(2 n+2 \alpha-2 j) !}{j !(n+\alpha-j) !} \frac{u^{n-2 j}}{(n-2 j) !},
$$

usually found in other indirect way. A direct proof of this evaluation is given in Appendix B. ${ }^{3}$ Using the duplication formula for the argument of the Gamma function one finds from (4.4) the equivalent representation

$$
\begin{aligned}
& P_{n}^{(\alpha, \alpha)}(u)=\frac{2^{2 \alpha}(n+\alpha) !}{(n+2 \alpha) !\left(-\frac{1}{2}\right) !} \sum_{j=0}^{\left[\frac{n}{2}\right]} \frac{(-1)^{j}\left(n+\alpha-j-\frac{1}{2}\right) !}{j !(n-2 j) !}(2 u)^{n-2 j}, \\
& \left(-\frac{1}{2}\right) !=\sqrt{\pi} .
\end{aligned}
$$

Thus we obtained the two equivalent basic representations of the Ultraspherical polynomials $P_{n}^{(\alpha, \alpha)}(u)$ by an expansion in powers of the variable $u$ that was not possible in such simple way for the general Jacobi polynomials $P^{(\alpha, \beta)}(u)$.

From (4.4) follows for the argument $u=0$ of $P_{n}^{(\alpha, \alpha)}(u)$ for even $n=2 m$ and odd $n=2 m+1$

$$
P_{2 m}^{(\alpha, \alpha)}(0)=\frac{(-1)^{m}(2 m+\alpha) !}{2^{2 m} m !(m+\alpha) !}, \quad P_{2 m+1}^{(\alpha, \alpha)}(0)=0,
$$

where the vanishing for odd $n$ is due to the symmetry (3.4). If we write down the expansion (2.12) in special case $\alpha=\beta$ we have after changing the index of summation according to $k \leftrightarrow n-l, l \leftrightarrow n-k$

$$
P_{n}^{(\alpha, \alpha)}(u)=\sum_{l=0}^{n} \frac{(2 n+2 \alpha-l) !}{2^{n-l}(n-l) !(n+2 \alpha) !} P_{l}^{(n+\alpha-l, n+\alpha-l)}(0) u^{n-l},
$$

and by comparison of (4.4) with (5.2) or directly from (4.6) we find

$$
(-1)^{j} 2^{2 j} P_{2 j}^{(n+\alpha-2 j, n+\alpha-2 j)}(0)=\frac{(n+\alpha) !}{j !(n+\alpha+j) !}, \quad P_{2 j+1}^{(n+\alpha-2 j-1, n+\alpha-2 j-1)}(0)=0 .
$$

The expression for $(-1)^{j} 2^{2 j} P_{2 j}^{(n+\alpha-2 j, n+\alpha-2 j)}(0)$ is a binomial coefficient $\left(\begin{array}{c}n+\alpha \\ j\end{array}\right)$ (or its generalization for non-integer $\alpha$ ). It is interesting that we met already the case $\alpha=0$ when considering the expansion of $(x+i y)^{n}(x-i y)^{n}=\left(x^{2}+y^{2}\right)^{n}$ in powers of products of $x$ and $y$ and the ${ }^{3}$ Although the obtained explicit representation of the Ultraspherical polynomials (4.4) is known from other more indirect approaches it is interesting to make this sum evaluation directly in (4.3), moreover, since in next Section we encounter another sum which can be evaluated in similar way. 
representation of the coefficients by the Jacobi polynomials as special case $m=n$ of $(x+i y)^{m}(x-i y)^{n} \quad$ [40] (and papers cited therein).

\section{Alternative Representation of Ultraspherical Polynomials}

We derive in this Section an alternative explicit representation of the Ultraspherical polynomials which becomes important for our further considerations.

We start from the representation (4.4) of $P_{n}^{(\alpha, \alpha)}(u)$ which we write in a slightly different form and make the following transformations using the binomial formula and reordering of the arising double sum

$$
\begin{aligned}
& P_{n}^{(\alpha, \alpha)}(u) \\
= & \frac{(n+\alpha) !}{(n+2 \alpha) !}\left(\frac{u}{2}\right)^{n} \sum_{j=0}^{\left.n \frac{n}{2}\right]} \frac{(-1)^{j}(2 n+2 \alpha-2 j) !}{j !(n-2 j) !(n+\alpha-j) !}\left(\frac{1-u^{2}}{u^{2}}+1\right)^{j} \\
= & \frac{(n+\alpha) !}{(n+2 \alpha) !}\left(\frac{u}{2}\right)^{n} \sum_{j=0}^{\left.n \frac{n}{2}\right]} \frac{(-1)^{j}(2 n+2 \alpha-2 j) !}{j !(n-2 j) !(n+\alpha-j) !} \sum_{k=0}^{j} \frac{j !}{k !(j-k) !}\left(\frac{1-u^{2}}{u^{2}}\right)^{k} \\
= & \frac{(n+\alpha) !}{(n+2 \alpha) !}\left(\frac{u}{2}\right)^{n} \sum_{k=0}^{\left.n \frac{n}{2}\right]} \frac{1}{k !}\left(\sum_{j=0}^{\left[\frac{n}{2}\right]} \frac{(-1)^{j}(2 n+2 \alpha-2 j) !}{(n-2 j) !(n+\alpha-j) !(j-k) !}\right)\left(\frac{1-u^{2}}{u^{2}}\right)^{k} .
\end{aligned}
$$

With the evaluation of the inner sum which we make in detail in Appendix B we obtain the following substantial new representation of the Ultraspherical polynomials

$$
P_{n}^{(\alpha, \alpha)}(u)=\sum_{k=0}^{\left[\frac{n}{2}\right]} \frac{(-1)^{k}(n+\alpha) !}{k !(n-2 k) !(k+\alpha) ! 2^{2 k}}\left(1-u^{2}\right)^{k} u^{n-2 k} .
$$

One can also make the inverse transition from this representation to the representation (4.4) by expansion of $\left(1-u^{2}\right)^{k}$ into a Taylor series in powers of $u^{2}$ and evaluation of the arising (inner) sum.

We make now the following coordinate transformation $u \leftrightarrow x$

$$
\begin{aligned}
& u=\frac{x}{\sqrt{1+x^{2}}}, x=\frac{u}{\sqrt{1-u^{2}}},\left(1-u^{2}\right)\left(1+x^{2}\right)=1, \\
& \frac{\partial u}{\partial x}=\frac{1}{\left(1+x^{2}\right)^{\frac{3}{2}}}, \frac{\partial x}{\partial u}=\frac{1}{\left(1-u^{2}\right)^{\frac{3}{2}}}, \\
& \mathrm{~d} u=\frac{\partial u}{\partial x} \mathrm{~d} x, \mathrm{~d} x=\frac{\partial x}{\partial u} \mathrm{~d} u, \frac{\partial}{\partial u}=\frac{\partial x}{\partial u} \frac{\partial}{\partial x}, \frac{\partial}{\partial x}=\frac{\partial u}{\partial x} \frac{\partial}{\partial u} .
\end{aligned}
$$

The variable $u$ in the interval $[-1,+1]$ is here extended to the whole real axis $(-\infty,+\infty)$ for the variable $x$.

If we make this substitution of the variable $u$ in (2) according to (3) we find the following relation

$$
P_{n}^{(\alpha, \alpha)}\left(\frac{x}{\sqrt{1+x^{2}}}\right)=\frac{1}{\left(\sqrt{1+x^{2}}\right)^{n}} \sum_{k=0}^{\left[\frac{n}{2}\right.} \frac{(-1)^{k}(n+\alpha) !}{k !(k+\alpha) !(n-2 k) !} \frac{x^{n-2 k}}{2^{2 k}} .
$$


Therefore $\left(\sqrt{1+x^{2}}\right)^{n} P_{n}^{(\alpha, \alpha)}\left(\frac{x}{\sqrt{1+x^{2}}}\right)$ is a polynomial of the variable $x$ of degree $n$ and we introduce for it multiplied by a factor for practical purpose a new notation $G_{n}^{\alpha}(x)$ according to

$$
\begin{aligned}
G_{n}^{\alpha}(x) & \equiv \frac{n ! \alpha !}{(n+\alpha) !}\left(\sqrt{1+x^{2}}\right)^{n} P_{n}^{(\alpha, \alpha)}\left(\frac{x}{\sqrt{1+x^{2}}}\right) \\
& =\sum_{k=0}^{\left[\frac{n}{2}\right]} \frac{(-1)^{k} \alpha ! n !}{k !(k+\alpha) !(n-2 k) !} \frac{x^{n-2 k}}{2^{2 k}},
\end{aligned}
$$

or in representation by the Hypergeometric function ${ }_{2} F_{1}(a, b ; c ; z)$

$$
G_{n}^{\alpha}(x)=x_{2}^{n} F_{1}\left(-\frac{n}{2},-\frac{n-1}{2} ; \alpha+1 ;-\frac{1}{x^{2}}\right) \text {. }
$$

In Appendix $\mathrm{C}$ we give tables of the polynomials $G_{n}^{\alpha}(x)$ for the special cases $\alpha=-\frac{1}{2}, 0, \frac{1}{2}$.

Formula (5.4) can be represented in the form

$$
\begin{aligned}
G_{n}^{\alpha}(x) & =\sum_{k=0}^{\left[\frac{n}{2}\right]} \frac{(-1)^{k} \alpha !}{k !(k+\alpha) !}\left(\frac{1}{2} \frac{\partial}{\partial x}\right)^{2 k} x^{n} \\
& =\left\{1-\frac{\alpha !}{1 !(1+\alpha) !} \frac{1}{2^{2}} \frac{\partial^{2}}{\partial x^{2}}+\frac{\alpha !}{2 !(2+\alpha) !} \frac{1}{2^{4}} \frac{\partial^{4}}{\partial x^{4}}-\cdots\right\} x^{n},
\end{aligned}
$$

or if we use the standard notation for the Bessel functions $J_{\alpha}(z)$ and their well-known Taylor series

$$
G_{n}^{\alpha}(x)=\alpha ! \frac{J_{\alpha}\left(\frac{\partial}{\partial x}\right)}{\left(\frac{1}{2} \frac{\partial}{\partial x}\right)^{\alpha}} x^{n} \equiv j_{\alpha}\left(\frac{\partial}{\partial x}\right) x^{n}, \quad G_{0}^{\alpha}(x)=1 .
$$

We have now obtained a very interesting formula for the polynomials $G_{n}^{\alpha}(x)$, $(n, 0,1,2, \cdots)$. They can be obtained by application of the (integral) operator

$$
j_{\alpha}\left(\frac{\partial}{\partial x}\right)=\alpha ! \frac{J_{\alpha}\left(\frac{\partial}{\partial x}\right)}{\left(\frac{1}{2} \frac{\partial}{\partial x}\right)^{\alpha}}, \leftrightarrow j_{\alpha}(y) \equiv \alpha ! \frac{J_{\alpha}(y)}{\left(\frac{y}{2}\right)^{\alpha}},\left(y \leftrightarrow \frac{\partial}{\partial x}\right),
$$

onto the monomials $x^{n}$. Although this operator depends on $\alpha$ as a fixed parameter it does not depend on the degree $n$ of the polynomial ${ }^{4}$.

${ }^{4}$ This is in great analogy to the Hermite polynomials $H_{n}(x)$ which can be generated from the monomials $x^{n}$ by the formula [35] [40]

$$
H_{n}(x)=\exp \left(-\frac{1}{4} \frac{\partial^{2}}{\partial x^{2}}\right)(2 x)^{n}
$$

as an alternative definition and which in some of our paper of the last years plaid an important role. The oldest source for this formula which we found up to now, however, without a citation there is the book of Bell [22] (p. 159). 
From the differential Equation (3.8) for $P_{n}^{(\alpha, \alpha)}(u)$ follows via the transformation (5.3) the differential equation for $G_{n}^{\alpha}(x)$

$$
\left\{\frac{\partial}{\partial x}\left(1+x^{2}\right) \frac{\partial}{\partial x}-(2 n+2 \alpha+1) x \frac{\partial}{\partial x}+n(n+2 \alpha)\right\} G_{n}^{\alpha}(x)=0 .
$$

They are not orthogonal polynomials as we will see. Instead of the polynomials $G_{n}^{\alpha}(x)$ we introduce now as intermediate step to orthonormalized functions the functions $G_{n}^{\prime \alpha}(x)$ by means of a weight function $w^{\prime}(x)$ as follows

$$
G_{n}^{\prime \alpha}(x) \equiv\left(1+x^{2}\right)^{-\left(\frac{n+\alpha}{2}+\frac{1}{4}\right)} G_{n}^{\alpha}(x), \quad w^{\prime}(x) \equiv \frac{1}{1+x^{2}} .
$$

From (5.10) follows that they obey the following differential equation with a self-adjoint operator in front

$$
\left\{\frac{\partial}{\partial x}\left(1+x^{2}\right) \frac{\partial}{\partial x}+\frac{(2 n+2 \alpha+1)^{2}}{4\left(1+x^{2}\right)}+\frac{1}{4}-\alpha^{2}\right\} G_{n}^{\prime \alpha}(x)=0 .
$$

In addition we define now the following set of functions $g_{n}^{\alpha}(x)$ by

$$
\begin{aligned}
g_{n}^{\alpha}(x) & \equiv \frac{1}{\alpha !} \sqrt{\frac{2 n+2 \alpha+1}{2^{2 \alpha+1}} \frac{(n+2 \alpha) !}{n !}}\left(1+x^{2}\right)^{-\left(\frac{n+\alpha}{2}+\frac{3}{4}\right)} G_{n}^{\alpha}(x) \\
& =\frac{1}{\alpha !} \sqrt{\frac{2 n+2 \alpha+1}{2^{2 \alpha+1}} \frac{(n+2 \alpha) !}{n !}} \sqrt{w^{\prime}(x)} G_{n}^{\prime \alpha}(x) .
\end{aligned}
$$

They satisfy the following differential equation

$$
\left\{\frac{\partial}{\partial x}\left(1+x^{2}\right)^{2} \frac{\partial}{\partial x}+\left(\frac{9}{4}-\alpha^{2}\right) x^{2}+\frac{(2 n+1)(2 n+4 \alpha+1)+5}{4}\right\} g_{n}^{\alpha}(x)=0 .
$$

From (3.12) follows their orthonormality

$$
\int_{-\infty}^{+\infty} \mathrm{d} x g_{m}^{\alpha}(x) g_{n}^{\alpha}(x)=\delta_{m, n},(\alpha>-1),
$$

and connected with them the completeness relations on the real axis

$$
\sum_{n=0}^{\infty} g_{n}^{\alpha}(x) g_{n}^{\alpha}(y)=\delta(x-y),(\alpha>-1) .
$$

After these preparations it is necessary here to mention the following. The sets of polynomials $G_{n}^{\alpha}(x)$ with fixed parameter $\alpha$ are not sets of Orthogonal polynomials in the usual sense because for their definition we cannot find a weight function $w^{\alpha}(x)$ which does not depend on the degree $n$ of the polynomials but may depend only on $\alpha$ as parameter but instead of this the sets of functions $G_{n}^{\prime \alpha}(x)$ are sets of orthogonal functions over the whole real axis to a weight $w^{\prime}(x)=\left(1+x^{2}\right)^{-1}$ which is independent on $\alpha$. Thus the sets of polynomials $G_{n}^{\alpha}(x)$ do not belong to the Classical orthogonal polynomials but are relatives to the Ultraspherical polynomials $P_{n}^{(\alpha, \alpha)}(u)$.

\section{Investigation of the Relatives to the Ultraspherical Polynomials and a Generating Function}

We continue in this section the investigation of the relatives $G_{n}^{\alpha}(x)$ to the 
Ultraspherical polynomials $P_{n}^{(\alpha, \alpha)}(u)$ defined by the operational relation (5.8)

$$
\left.G_{n}^{\alpha}(x)=j_{\alpha}\left(\frac{\partial}{\partial x}\right) x^{n}, \quad j_{\alpha}(y) \equiv \frac{\alpha ! J_{\alpha}(y)}{\left(\frac{y}{2}\right)^{\alpha}}\right),
$$

and with the explicit representation

$$
G_{n}^{\alpha}(x) \equiv \frac{\alpha !}{2^{n}} \sum_{k=0}^{\left[\frac{n}{2}\right]} \frac{(-1)^{k} n !}{k !(k+\alpha) !(n-2 k) !}(2 x)^{n-2 k} .
$$

Immediately we see that the powers $x^{n}$ of the variable $x$ can be represented (analogously to the case of Hermite polynomials) by application of the reciprocal operator to $j_{\alpha}(x)$ according to

$$
\begin{aligned}
x^{n} & =\left(j_{\alpha}\left(\frac{\partial}{\partial x}\right)\right)^{-1} G_{n}^{\alpha}(x)=\frac{\left(\frac{1}{2} \frac{\partial}{\partial x}\right)^{n}}{\alpha ! J_{\alpha}\left(\frac{\partial}{\partial x}\right)} G_{n}^{\alpha}(x) \\
& =\left\{1+\frac{\alpha !}{1 !(\alpha+1) !}\left(\frac{1}{2} \frac{\partial}{\partial x}\right)^{2}+\frac{\alpha !}{2 !(\alpha+2) !} \frac{\alpha+3}{\alpha+1}\left(\frac{1}{2} \frac{\partial}{\partial x}\right)^{4}+\cdots\right\} G_{n}^{\alpha}(x) .
\end{aligned}
$$

The reciprocal functions to $j_{\alpha}(x)$ are not explicitly considered up to now in the common literature and thus the practical use of the inversion (6.3) is limited.

For the further investigations it is useful to prepare a few relations for the introduced function $j_{\alpha}(x)$. From the recurrence relation of the Bessel functions $J_{\alpha}(x)$ and their differentiation formula follows translated to the function $j_{\alpha}(x)$

$$
j_{\alpha}(x)=j_{\alpha-1}(x)+\frac{x^{2}}{4(\alpha+1) \alpha} j_{\alpha+1}(x), \quad \frac{\partial j_{\alpha}}{\partial x}(x)=-\frac{x}{2(\alpha+1)} j_{\alpha+1}(x) .
$$

From the first relation one obtains the recurrence relation

$$
\begin{aligned}
G_{n}^{\alpha}(x) & =j_{\alpha}\left(\frac{\partial}{\partial x}\right) x^{n}=\left(j_{\alpha-1}\left(\frac{\partial}{\partial x}\right)+\frac{1}{4(\alpha+1) \alpha} \frac{\partial^{2}}{\partial x^{2}} j_{\alpha+1}\left(\frac{\partial}{\partial x}\right)\right) x^{n} \\
& =G_{n}^{\alpha-1}(x)+\frac{n(n-1)}{4(\alpha+1) \alpha} G_{n-2}^{\alpha+1}(x) .
\end{aligned}
$$

Represented as on operator identity with $y \rightarrow \frac{\partial}{\partial x}$ and $\frac{\partial}{\partial y} \rightarrow-x$ as an operator the second relation for the derivative of $j_{\alpha}(y)$ has to be written

$$
\frac{\partial}{\partial y} j_{\alpha}(y)=j_{\alpha}(y) \frac{\partial}{\partial y}-\frac{y}{2(\alpha+1)} j_{\alpha+1}(y) .
$$

Since in the commutation relation $1=\left[\frac{\partial}{\partial x}, x\right]=-\left[y, \frac{\partial}{\partial y}\right]$ of the multiplication operator $x$ with the differentiation operator $\frac{\partial}{\partial x}$ the commutator changes 
its sign exchanging $\left(y \leftrightarrow \frac{\partial}{\partial x}, \frac{\partial}{\partial y} \leftrightarrow x\right)$ we have interchanging these operators but preserving the commutator only to change the sign of one of these operators, for example $\left(x \rightarrow \frac{\partial}{\partial x}, \frac{\partial}{\partial x} \rightarrow-x\right)$ and the operator identity (6.6) can be translated into the operator identity

$$
x j_{\alpha}\left(\frac{\partial}{\partial x}\right)=j_{\alpha}\left(\frac{\partial}{\partial x}\right) x+\frac{1}{2(\alpha+1)} \frac{\partial}{\partial x} j_{\alpha+1}\left(\frac{\partial}{\partial x}\right) .
$$

Operator identities can be applied to arbitrary functions (here $f(x)$ ) to get correct results in every case.

The result of the differentiation of the polynomials $G_{n}^{\alpha}(x)$ is easily to see

$$
\frac{\partial}{\partial x} G_{n}^{\alpha}(x)=\frac{\partial}{\partial x} j_{\alpha}\left(\frac{\partial}{\partial x}\right) x^{n}=n j_{\alpha}\left(\frac{\partial}{\partial x}\right) x^{n-1}=n G_{n-1}^{\alpha}(x) .
$$

Therefore, the operator $\frac{\partial}{\partial x}$ is the lowering operator for the sets of polynomials $G_{n}^{\alpha}(x)$.

The case of the raising operator is more difficult and we delay this problem to Section 9 where we describe the $S U(1,1)$ symmetry of the of the Ultraspherical polynomials $P_{n}^{(\alpha, \alpha)}(u)$ and of the polynomials $G_{n}^{\alpha}(x)$. However, applying, for example, the operator identity (6.7) we obtain a partially interesting result

$$
\begin{aligned}
x G_{n}^{\alpha}(x) & =x j_{n}^{\alpha}\left(\frac{\partial}{\partial x}\right) x^{n}=\left\{j_{\alpha}\left(\frac{\partial}{\partial x}\right) x x^{n}+\frac{1}{2(\alpha+1)} j_{\alpha+1}\left(\frac{\partial}{\partial x}\right) \frac{\partial}{\partial x} x^{n}\right\} \\
& =G_{n+1}^{\alpha}(x)+\frac{n}{2(\alpha+1)} G_{n-1}^{\alpha+1}(x) .
\end{aligned}
$$

This is not a pure raising relation for the polynomials $G_{n}^{\alpha}(x)$ since in the second part on the right-hand side we have yet a lowering of the index $n$ accompanied with the raising of the upper index $\alpha$.

Another approach to the problem of the raising operator leads only to a form which is formally dependent on the degree $n$ of the polynomial and we make this in Sections 8 and 9 for the normalized polynomials $g_{n}^{\alpha}(x)$ after the preparation of shortly considering general $S U(, 1)$-symmetry. From the differential Equation (5.10) for $G_{n}^{\alpha}(x)$ together with the lowering relation (6.8) follows

$$
\left(\frac{\partial}{\partial x}\left(1+x^{2}\right)-(2 n+2 \alpha+1) x\right) G_{n-1}^{\alpha}(x)+(n+2 \alpha) G_{n}^{\alpha}(x)=0,
$$

which raising the index $n \rightarrow n+1$ leads to

$$
\frac{1}{n+2 \alpha+1}\left((2 n+2 \alpha+1) x-\left(1+x^{2}\right) \frac{\partial}{\partial x}\right) G_{n}^{\alpha}(x)=G_{n+1}^{\alpha}(x) \text {. }
$$

The operator in front of $G_{n}^{\alpha}(x)$ on the left-hand side may be seen as a form of the creation operator, which however depends on the index $n$ of $G_{n}^{\alpha}$ to which it is applied and must be changed if it is applied to a polynomial with changed 
index $n$.

With the representation of the polynomials $G_{n}^{\alpha}(x)$ according to (6.1) one may calculate the following generating function in an easy way

$$
\begin{aligned}
\sum_{n=0}^{\infty} G_{n}^{\alpha}(x) \frac{t^{n}}{n !} & =j_{\alpha}\left(\frac{\partial}{\partial x}\right) \sum_{n=0}^{\infty} \frac{(x t)^{n}}{n !}=j_{\alpha}\left(\frac{\partial}{\partial x}\right) e^{x t} \\
& =e^{x t} j_{\alpha}\left(\frac{\partial}{\partial x}+t\right) 1=e^{x t} j_{\alpha}(t),
\end{aligned}
$$

that means with the definition of $j_{\alpha}(x)$ in (6.1)

$$
\sum_{n=0}^{\infty} G_{n}^{\alpha}(x) \frac{t^{n}}{n !}=\exp (x t) \frac{2^{\alpha} \alpha ! J_{\alpha}(t)}{t^{\alpha}} .
$$

We used the commutation relation

$$
e^{-x t} \frac{\partial}{\partial x} e^{x t}=\frac{\partial}{\partial x}+t
$$

The number of different generating functions which one may calculate in this way is not very large since usually it is not possible to get an explicit result if one applies the operator $j_{\alpha}\left(\frac{\partial}{\partial x}\right)$ to a sum $\sum_{n=0}^{\infty} c_{n}(x t)^{n}$ which last one often may not calculate. However, in cases if one has calculated a generating function in another way also for the Ultraspherical polynomials then one may establish a relation to the described way that is usually equivalent to find ordering relations for combinations of functions of operators $x$ and $\frac{\partial}{\partial x}$ which are difficult to find or to conjecture in another way.

\section{Back Transformation to the Ultraspherical Polynomials and Their Alternative Definition}

In this section we make the inverse transformation from the polynomials $G_{n}^{\alpha}(x)$ back to the Ultraspherical polynomials $P_{n}^{(\alpha, \alpha)}(u)$ from relation (5.5)

$$
\begin{aligned}
P_{n}^{(\alpha, \alpha)}\left(\frac{x}{\sqrt{1+x^{2}}}\right) & =\frac{(n+\alpha) !}{n ! \alpha !} \frac{1}{\left(\sqrt{1+x^{2}}\right)^{n}} G_{n}^{\alpha}(x) \\
& =\frac{(n+\alpha) !}{n ! \alpha !} \frac{1}{\left(\sqrt{1+x^{2}}\right)^{n}} j_{\alpha}\left(\frac{\partial}{\partial x}\right) x^{n} .
\end{aligned}
$$

If one goes now back from the coordinate $x$ to the coordinate $u$ by relations (5.3) one finds

$$
\begin{aligned}
P_{n}^{(\alpha, \alpha)}(u) & =\frac{(n+\alpha) !}{n ! \alpha !}\left(\sqrt{1-u^{2}}\right)^{n} j_{\alpha}\left(\left(\sqrt{1-u^{2}}\right)^{3} \frac{\partial}{\partial u}\right)\left(\frac{u}{\sqrt{1-u^{2}}}\right)^{n} \\
& =\frac{(n+\alpha) !}{n !}\left(\sqrt{1-u^{2}}\right)^{n} \frac{2^{\alpha} J_{\alpha}\left(\left(\sqrt{1-u^{2}}\right)^{3} \frac{\partial}{\partial u}\right)}{\left(\left(\sqrt{1-u^{2}}\right)^{3} \frac{\partial}{\partial u}\right)^{\alpha}}\left(\frac{u}{\sqrt{1-u^{2}}}\right)^{n} .
\end{aligned}
$$


This may be considered as an alternative definition of the Ultraspherical polynomials in comparison to the Rodrigues definition (3.3) (see footnote 2 in Section 5) which on the first glance seems to be awkward but if we look nearer to it we discover also some interesting features and representations, in particular, when we represent this by trigonometric or hyperbolic functions. It is interesting that only the fixed operator $j_{\alpha}(x)$ with $x \rightarrow\left(\sqrt{1-u^{2}}\right)^{3} \frac{\partial}{\partial u}$ which is independent of the degree $n$ of the polynomials plays a main role in application to the monomials of $x=\frac{u}{\sqrt{1-u^{2}}}$. Furthermore interesting in this definition is that the Bessel functions $J_{\alpha}(x)$ are closely related with the Ultraspherical polynomials $P_{n}^{(\alpha, \alpha)}(u)$.

If one tries to make the opposite to go from the Ultraspherical polynomials $P_{n}^{(\alpha, \alpha)}(u)$ back to the polynomials $G_{n}^{\alpha}(x)$ by relation (5.5) in coordinate $u$ and insert for $P_{n}^{(\alpha, \alpha)}(u)$ the Rodrigues definition (3.3)

$$
\begin{aligned}
G_{n}^{\alpha}\left(\frac{u}{\sqrt{1-u^{2}}}\right) & =\frac{n ! \alpha !}{(n+\alpha) !} \frac{1}{\left(\sqrt{1-u^{2}}\right)^{n}} P_{n}^{(\alpha, \alpha)}(u) \\
& =\frac{n ! \alpha !}{(n+\alpha) !} \frac{1}{\left(\sqrt{1-u^{2}}\right)^{n}} \frac{(-1)^{n}}{2^{n} n !} \frac{1}{\left(1-u^{2}\right)^{\alpha}} \frac{\partial^{n}}{\partial u^{n}}\left(1-u^{2}\right)^{n+\alpha},
\end{aligned}
$$

and make the transition from coordinate $u$ to the coordinate $x$ via relations (3) then one finds as the Rodrigues-like definition of $G_{n}^{\alpha}(x)$

$$
G_{n}^{\alpha}(x)=\frac{(-1)^{n} \alpha !}{2^{n}(n+\alpha) !}\left(\sqrt{1+x^{2}}\right)^{n+2 \alpha}\left(\left(\sqrt{1+x^{2}}\right)^{3} \frac{\partial}{\partial x}\right)^{n} \frac{1}{\left(1+x^{2}\right)^{n+\alpha}} .
$$

In comparison with the definition (6.1) this definition seems to be more complicated in its structure and the conjecture is that no other substantial different Rodrigues-like definition of the polynomials $G_{n}^{\alpha}(x)$ exists.

For the variable $u$ within the borders $-1 \leq u \leq+1$ we make now the substitution $u=\cos (\theta)$ and obtain from (7.2)

$$
P_{n}^{(\alpha, \alpha)}(\cos (\theta))=\frac{(n+\alpha) !}{n !} \sin ^{n}(\theta) \frac{2^{\alpha} J_{\alpha}\left(\sin ^{2}(\theta) \frac{\partial}{\partial \theta}\right)}{\left(\sin ^{2}(\theta) \frac{\partial}{\partial \theta}\right)^{\alpha}}\left(\frac{\cos (\theta)}{\sin (\theta)}\right)^{n} .
$$

For the use of the Ultraspherical polynomials with the variable $u$ in the range $|u| \geq 1$ we can make the substitution by the Hyperbolic Cosine $u=\operatorname{ch}(\chi)$ and obtain

$$
P_{n}^{(\alpha, \alpha)}(\operatorname{ch}(\chi))=\frac{(n+\alpha) !}{n !} \operatorname{sh}^{n}(\chi) \frac{2^{\alpha} I_{\alpha}\left(\operatorname{sh}^{2}(\chi) \frac{\partial}{\partial \chi}\right)}{\left(\operatorname{sh}^{2}(\chi) \frac{\partial}{\partial \chi}\right)^{\alpha}}\left(\frac{\operatorname{ch}(\chi)}{\operatorname{sh}(\chi)}\right)^{n} .
$$


In comparison, the Rodrigues definition (3.3) in variable $u=\cos (\theta)$ takes on the form

$$
P_{n}^{(\alpha, \alpha)}(\cos (\theta))=\frac{1}{2^{n} n !} \frac{1}{\sin ^{2 \alpha}(\theta)}\left(\frac{1}{\sin (\theta)} \frac{\partial}{\partial \theta}\right)^{n} \sin ^{2 n+2 \alpha}(\theta),
$$

and in variable $u=\operatorname{ch}(\chi)$

$$
P_{n}^{(\alpha, \alpha)}(\operatorname{ch}(\chi))=\frac{1}{2^{n} n !} \frac{1}{\operatorname{sh}^{2 \alpha}(\chi)}\left(\frac{1}{\operatorname{sh}(\chi)} \frac{\partial}{\partial \chi}\right)^{n} \operatorname{sh}^{2 n+2 \alpha}(\chi) .
$$

The powers of the operators $\frac{1}{\sin (\theta)} \frac{\partial}{\partial \theta}$ or $\frac{1}{\operatorname{sh}(\chi)} \frac{\partial}{\partial \chi}$ are not "disentangled" that means the powers of the differential operators $\frac{\partial}{\partial \theta}$ or $\frac{\partial}{\partial \chi}$ do not generally stand to the right of the multiplication operators that makes their application difficult.

Let us consider for convenience in greater detail the three special cases of $\alpha=-\frac{1}{2}, 0,+\frac{1}{2}$ for which the functions $j_{\alpha}(x)$ are

$$
j_{-\frac{1}{2}}(x)=\cos (x), j_{0}(x)=J_{0}(x), j_{+\frac{1}{2}}(x)=\frac{\sin (x)}{x} .
$$

They lead to the Chebyshev polynomials of first and second kind $T_{n}(u)$ and $U_{n}(u)$ and to the Legendre polynomials $P_{n}(u)$. The alternative definitions of the corresponding Ultraspherical polynomials are then

$$
\begin{aligned}
P_{n}^{\left(-\frac{1}{2},-\frac{1}{2}\right)}(\cos (\theta)) & =\frac{\left(n-\frac{1}{2}\right) !}{n !\left(-\frac{1}{2}\right) !} \sin ^{n}(\theta) \cos \left(\sin ^{2}(\theta) \frac{\partial}{\partial \theta}\right)\left(\frac{\cos (\theta)}{\sin (\theta)}\right)^{n} \\
& =\frac{\left(n-\frac{1}{2}\right) !}{n !\left(-\frac{1}{2}\right) !} T_{n}(\cos (\theta)), \\
P_{n}^{(0,0)}(\cos (\theta)) & =\sin ^{n}(\theta) J_{0}\left(\sin ^{2}(\theta) \frac{\partial}{\partial \theta}\right)\left(\frac{\cos (\theta)}{\sin (\theta)}\right)^{n}=P_{n}(\cos (\theta)), \\
P_{n}^{\left(\frac{1}{2}, \frac{1}{2}\right)}(\cos (\theta)) & =\frac{\left(n+\frac{1}{2}\right) !}{n !\left(\frac{1}{2}\right) !} \sin ^{n}(\theta) \frac{\sin ^{2}\left(\sin ^{2}(\theta) \frac{\partial}{\partial \theta}\right)}{\sin ^{2}(\theta) \frac{\partial}{\partial \theta}}\left(\frac{\cos (\theta)}{\sin (\theta)}\right)^{n} \\
& =\frac{\left(n+\frac{1}{2}\right) !}{n !\left(\frac{1}{2}\right) !} \frac{U_{n}(\cos (\theta))}{n+1} .
\end{aligned}
$$

in comparison to the Rodrigues definition 


$$
\begin{aligned}
& P_{n}^{\left(-\frac{1}{2},-\frac{1}{2}\right)}(\cos (\theta))=\frac{1}{2^{n} n !} \sin (\theta)\left(\frac{1}{\sin (\theta)} \frac{\partial}{\partial \theta}\right)^{n} \sin ^{2 n-1}(\theta) \\
& =\frac{\left(n-\frac{1}{2}\right) !}{n !\left(-\frac{1}{2}\right) !} T_{n}(\cos (\theta)), \\
& P_{n}^{(0,0)}(\cos (\theta))=\frac{1}{2^{n} n !}\left(\frac{1}{\sin (\theta)} \frac{\partial}{\partial \theta}\right)^{n} \sin ^{2 n}(\theta)=P_{n}(\cos (\theta)) \\
& P_{n}^{\left(\frac{1}{2}, \frac{1}{2}\right)}(\cos (\theta))=\frac{1}{2^{n} n !} \frac{1}{\sin (\theta)}\left(\frac{1}{\sin (\theta)} \frac{\partial}{\partial \theta}\right)^{n} \sin ^{2 n+1}(\theta) \\
& =\frac{\left(n+\frac{1}{2}\right) !}{n !\left(\frac{1}{2}\right) !} \frac{U_{n}(\cos (\theta))}{n+1} .
\end{aligned}
$$

One difficulty in last definitions as explained is that the operators $\left(\frac{1}{\sin (\theta)} \frac{\partial}{\partial \theta}\right)^{n}$ are not "disentangled" as pure series in the differentiation operator $\frac{\partial}{\partial \theta}$ and depend on the degree $n$ of the polynomials.

Here is the right place to mention the following known interesting relations [1] [3]

$$
\cos (n \theta)=T_{n}(\cos (\theta)), \frac{\sin ((n+1) \theta)}{\sin (\theta)}=U_{n}(\cos (\theta)),
$$

which can be proved, for example, by comparison of the expansions of $\cos (n \theta)$ and of $\frac{\sin ((n+1) \theta)}{\sin (\theta)}$ in powers of $\cos (\theta)$ with the explicit expressions for $T_{n}(u)$ and $U_{n}(u)$. For $P_{n}(\cos (\theta))$ such a simple relation to trigonometric functions is not existing but there is another interesting known case (see, e.g., [1], Equation $(4.1,8)$ or $[4])$

$$
\begin{aligned}
& \frac{\cos \left((2 n+1) \frac{\theta}{2}\right)}{\cos \left(\frac{\theta}{2}\right)}=\frac{2^{2 n} n !^{2}}{(2 n) !} P_{n}^{\left(-\frac{1}{2}, \frac{1}{2}\right)}(\cos (\theta)), \\
& \frac{\sin \left((2 n+1) \frac{\theta}{2}\right)}{\sin \left(\frac{\theta}{2}\right)}=\frac{2^{2 n} n !^{2}}{(2 n) !} P_{n}^{\left(\frac{1}{2},-\frac{1}{2}\right)}(\cos (\theta)),
\end{aligned}
$$

with such simple relations to the algebra of trigonometric functions but it does not belong to the Ultraspherical polynomials since their upper indices are not equal.

Let us now translate the generating function (13) for $G_{n}^{\alpha}(x)$ into a generating 
function for $P_{n}^{(\alpha, \alpha)}(u)$ that leads immediately to (we change temporarily variable $\left.t \rightarrow t^{\prime}\right)$

$$
\sum_{n=0}^{\infty} \frac{n ! \alpha !}{(n+\alpha) !}\left(\sqrt{1+x^{2}}\right)^{n} P_{n}^{(\alpha, \alpha)}\left(\frac{x}{\sqrt{1+x^{2}}}\right) \frac{t^{\prime n}}{n !}=\exp \left(t^{\prime} x\right) \frac{2^{\alpha} \alpha ! J_{\alpha}\left(t^{\prime}\right)}{t^{\prime \alpha}} .
$$

After transformation to the variable $u$ according to (5.3) one finds

$$
\sum_{n=0}^{\infty} \frac{1}{(n+\alpha) !} P_{n}^{(\alpha, \alpha)}(u)\left(\frac{t^{\prime}}{\sqrt{1-u^{2}}}\right)^{n}=\exp \left(\frac{t^{\prime} u}{\sqrt{1-u^{2}}}\right) \frac{2^{\alpha} J_{\alpha}\left(t^{\prime}\right)}{t^{\prime \alpha}},
$$

that after the substitution $t^{\prime}=t \sqrt{1-u^{2}}$ is equivalent to

$$
\begin{aligned}
\sum_{n=0}^{\infty} P_{n}^{(\alpha, \alpha)}(u) \frac{t^{n}}{(n+\alpha) !} & =\exp (t u) \frac{2^{\alpha} J_{\alpha}\left(t \sqrt{1-u^{2}}\right)}{\left(t \sqrt{1-u^{2}}\right)^{\alpha}}=\exp (t u) \frac{j_{\alpha}\left(t \sqrt{1-u^{2}}\right)}{\alpha !} \\
& =\exp (t u) \sum_{l=0}^{\infty} \frac{1}{l !}\left(\frac{t}{2}\right)^{2 l} \frac{J_{l+\alpha}(t)}{\left(\frac{t}{2}\right)^{l+\alpha}} u^{2 l} \\
& =\exp (t u) \sum_{l=0}^{\infty} \frac{1}{l !}\left(\frac{t}{2}\right)^{l-\alpha} J_{l+\alpha}(t) u^{2 l},
\end{aligned}
$$

where in second line we made a Taylor series expansion of $j_{\alpha}\left(t \sqrt{1-u^{2}}\right)$ in powers of $u^{2}$. Setting $u=1$ in this relation and using $P_{n}^{(\alpha, \beta)}(1)=\frac{(n+\alpha) !}{n ! \alpha !}$ (see (2.5)) one finds the interesting identity

$$
\frac{1}{\alpha !}=\sum_{l=0}^{\infty} \frac{1}{l !}\left(\frac{t}{2}\right)^{l-\alpha} J_{l+\alpha}(t)
$$

with $t$ as an arbitrary complex parameter.

In the special cases $\alpha=-\frac{1}{2}, 0, \frac{1}{2}$ this generating function takes on the special forms

$$
\begin{aligned}
& \sum_{n=0}^{\infty} T_{n}(u) \frac{t^{n}}{n !}=\exp (t u) \cos \left(t \sqrt{1-u^{2}}\right) \\
& =\exp (t u) \sqrt{\pi} \sum_{l=0}^{\infty} \frac{1}{l !}\left(\frac{t}{2}\right)^{l+\frac{1}{2}} J_{l-\frac{1}{2}}(t) u^{2 l}, \\
& \sum_{n=0}^{\infty} P_{n}(u) \frac{t^{n}}{n !}=\exp (t u) J_{0}\left(t \sqrt{1-u^{2}}\right) \\
& =\exp (t u) \sum_{l=0}^{\infty} \frac{1}{l !}\left(\frac{t}{2}\right)^{l} J_{l}(t) u^{2 l}, \\
& \sum_{n=0}^{\infty} U_{n}(u) \frac{t^{n}}{(n+1) !}=\exp (t u) \frac{\sin \left(t \sqrt{1-u^{2}}\right)}{t \sqrt{1-u^{2}}} \\
& =\exp (t u) \frac{\sqrt{\pi}}{2} \sum_{l=0}^{\infty} \frac{1}{l !}\left(\frac{t}{2}\right)^{l-\frac{1}{2}} J_{l+\frac{1}{2}}(t) u^{2 l} .
\end{aligned}
$$


At least the second of these generating functions for the Legendre polynomials $P_{n}(u)$ is known (see [10], p. 165, Equation (5)) but it does not belong to the best-known generating functions and is rarely considered in the corresponding literature.

\section{Unitary Irreducible Representations of Lie Algebra su $(1,1)$}

In [41] it was proved in a very general way that a quantum-mechanical system with a quadratic spectrum of the energy levels $E_{n}$ as function of $n$ ordered by arising energy possesses a Lie algebra $s u(1,1)$ of lowering and raising operators whereas a linear spectrum as function of $n$ belongs to a Heisenberg-Weyl algebra $w(2)$ (two-dimensional phase space) as it is well known from the harmonic oscillator. If by changing a parameter of the system one has a limiting transition from a quadratic spectrum to a linear spectrum of energy eigenvalues then this transition is expressed by a limiting transition of the corresponding Lie algebras from $s u(1,1)$ to $w(2)$. Such a transition is called Inönü-Wigner contraction (see [41]). This procedure is applicable to the Ultraspherical polynomials and their relatives and the orthonormalized functions determined by them. We may see this from the differential equation for the Ultraspherical polynomials $P_{n}^{(\alpha, \alpha)}(u)$ in (3.8) or for $\sqrt{w^{(\alpha, \alpha)}} P^{(\alpha, \alpha)}(u)$ in (3.9) which last leads to the orthonormalized functions $p_{n}^{(\alpha, \alpha)}(u)$ where the part with the dependence on $n$ is a quadratic one.

With the parts which are independent on $n$ in these differential equations one may determine an analogue to the quantum-mechanical Hamilton operator in the Schrödinger equation. The same is true for the discussed relatives $G_{n}^{\alpha}(x)$ of the Ultraspherical polynomials and to their orthonormalized variants $g_{n}^{\alpha}(x)$ where in their differential Equations (5.10) for $G_{n}^{\alpha}(x)$ and (5.14) for $g_{n}^{\alpha}(x)$ the dependence on $n$ is a quadratical one. The explicit form of the operators of lowering and raising the indices in their concrete realization depends on the definition of the functions as normalized functions or as otherwise ones and only for the orthonormalized functions they can be defined in an abstract general form because all other forms are hardly to characterize by some common properties. This abstract form for the discrete representations of the Lie algebra $s u(1,1)$ to the Lie group $S U(1,1)$ was made in [42] and was given in many of our later papers. To prepare the special treatment for the Ultraspherical polynomials and for their relatives we compile first shortly and without proof the relations in the abstract form.

The Lie algebra $s u(1,1)$ is a real Lie algebra and possesses as basis three (by definition) Hermitean or self-adjoint basis operators $\left(K_{1}, K_{2}, K_{0}\right)$ which are usually determined in abstract way by the commutation relations

$$
\left[K_{1}, K_{2}\right]=-i K_{0}, \quad\left[K_{2}, K_{0}\right]=i K_{1}, \quad\left[K_{0}, K_{1}\right]=i K_{2}, \quad\left(K_{i}=K_{i}^{\dagger}, i=0,1,2\right) .
$$

Instead of $K_{1}$ and $K_{2}$ one may introduce the lowering operator $K_{-}$and the raising operator $K_{+}$by 


$$
K_{-} \equiv K_{1}-i K_{2}, \quad K_{+} \equiv K_{1}+i K_{2}, \quad\left(K_{-}^{\dagger}=K_{+}, K_{+}^{\dagger}=K_{-}\right)
$$

They obey the commutation relations

$$
\left[K_{-}, K_{+}\right]=2 K_{0},\left[K_{0}, K_{-}\right]=-K_{-},\left[K_{0}, K_{+}\right]=+K_{+} .
$$

By the substitutions $K_{1} \leftrightarrow i L_{1}, K_{2} \leftrightarrow i L_{2}, K_{0} \leftrightarrow L_{3}$ one obtains the commutation relations $\left[L_{i}, L_{j}\right]=i \epsilon_{i j k} L_{k}$ of the Lie algebra $s u(2)$ to the Lie group $S U$ (2) but this violates the Hermitecity of the operators $\left(L_{1}, L_{2}, L_{3}\right)$ if it is supposed for $\left(K_{1}, K_{2}, K_{0}\right)$ or vice versa. However, within the complex algebra $s l(2, \mathbb{C})$ to the Special complex linear group $S L(2, \mathbb{C})$ both real Lie algebras $s u(2)$ and $s u(1,1)$ are imbedded. An essential difference between $s u(2)$ and $s u(1,1)$ is that all unitary irreducible representations $D^{l}$ of $s u(2)$ are finite-dimensional with possible dimensions $n=2 l+1=0,1,2, \cdots, l=0, \frac{1}{2}, 1, \frac{3}{2}, \cdots$ whereas all unitary irreducible representations of $s u(1,1)$ are (countably) infinite-dimensional with real parameter $k$ which may take on continuous values in a certain range as we will see. One of the first and at once most comprehensive representations of the connection of Special functions to Lie algebras and Lie groups was given by Vilenkin [26] but it is not fully easy to establish the connection to our further representation here and in next Section ${ }^{5}$.

We now discuss in abstract form the unitary infinite-dimensional irreducible representations of $S U(1,1)$. Only under the very general supposition that there exists a normalized eigenstate $|k, 0\rangle$ of the operator $K_{0}$ to a real eigenvalue $k$ which is annihilated by the lowering operator $K_{-}$according to

$$
K_{0}|k, 0\rangle=k|k, 0\rangle, \quad\langle k, 0 \mid k, 0\rangle=1, K_{-}|k, 0\rangle=0,
$$

where $k$ is a fixed parameter of the representation one may construct the whole algebra of $s u(1,1)$ in application to all eigenstates of this (irreducible) representation. One easily sees that the operator $K_{-}$lowers the eigenvalues by a step of 1 and $K_{+}$raises the eigenvalues of states by a step of 1 . We get in this way new eigenstates $|k, n\rangle$ of $K_{0}$ to eigenvalues $n+k$ according to

$$
K_{0}|k, n\rangle=(n+k)|k, n\rangle \text {, }
$$

${ }^{5}$ Vilenkin ([26], p. 133) works almost exceptionally with a set of functions $P_{m n}^{l}(z)$ instead of the Jacobi polynomials $P_{n}^{(\alpha, \beta)}(z)$ but widely equivalent to them and defined by

$$
P_{m n}^{l}(z) \equiv \frac{i^{m-n}}{2^{m}} \sqrt{\frac{(l-m) !(l+m) !}{(l-n) !(l+n) !}}(1-z)^{\frac{m-n}{2}}(1+z)^{\frac{m+n}{2}} P_{l-m}^{(m-n, m+n)}(z), \quad\left(m=\frac{\beta+\alpha}{2}, n=\frac{\beta-\alpha}{2}\right) .
$$

These functions are chosen as favorable ones for the representation of the finite-dimensional unitary representations of the Lie group $S U(2)$ following Gel'fand, Naimark et al. [27]. The finitedimensional representations of $S U(2)$ become non-unitary (or, more specifically, "quasi-unitary" according to used notion in [26]) for the transition from $S U(2)$ to $S U(1,1)$ by the mentioned substitutions and Vilenkin denotes this group by $Q U(2)$. The functions $P_{m n}^{l}(z)$ are then modified to functions with Gothic letter $P$ instead of Latin $P$ correspondingly to the transition $S U(2) \rightarrow Q U(2) \sim S U(1,1)$. 
which are orthogonal to each other for different $n$ and if we orthonormalize these states according to $\langle k, m \mid k, n\rangle=\delta_{m, n}$ the whole algebra $s u(1,1)$ takes on the following form in the realization by the abstract states $|k, n\rangle$ [42]

$$
\begin{aligned}
& K_{-}|k, n\rangle=\sqrt{n(n+2 k-1)}|k, n-1\rangle, \\
& K_{+}|k, n\rangle=\sqrt{(n+1)(n+2 k)}|k, n+1\rangle, \\
& K_{0}|k, n\rangle=(n+k)|k, n\rangle,\langle k, m \mid k, n\rangle=\delta_{m, n} .
\end{aligned}
$$

This means that the states $|k, n\rangle$ can be generated from the lowest state $|k, 0\rangle$ according to

$$
\begin{aligned}
& |k, n\rangle=\sqrt{\frac{(2 k-1) !}{n !(n+2 k-1) !}} K_{+}^{n}|k, 0\rangle, \\
& \Leftrightarrow\langle k, n|=\sqrt{\frac{(2 k-1) !}{n !(n+2 k-1) !}}\langle k, 0| K_{-}^{n} .
\end{aligned}
$$

By construction the unitary irreducible representations with parameter $k$ terminate to below with the state to $n=0$. Formulae (6) agree with corresponding formulae of Van der Jeugt [32] ((1.11) on p. 33).

From (8.6) one may derive the relations

$$
\begin{aligned}
& K_{-} K_{+}|k, n\rangle=(n+1)(n+2 k)|k, n\rangle, \\
& K_{+} K_{-}|k, n\rangle=n(n-1+2 k)|k, n\rangle .
\end{aligned}
$$

For the sum and difference of the operator products $K_{-} K_{+}$and $K_{+} K_{-}$one finds

$$
\begin{aligned}
& \frac{1}{2}\left(K_{-} K_{+}+K_{+} K_{-}\right)|k, n\rangle=\left(n^{2}+2 k n+k\right)|k, n\rangle, \\
& \frac{1}{2}\left(K_{-} K_{+}-K_{+} K_{-}\right)|k, n\rangle=(n+k)|k, n\rangle=K_{0}|k, n\rangle .
\end{aligned}
$$

The operator $C$ defined by

$$
\begin{aligned}
C & \equiv K_{0}^{2}-K_{1}^{2}-K_{2}^{2}=K_{0}^{2}-\frac{1}{2}\left(K_{+} K_{-}+K_{-} K_{+}\right) \\
& =k(k-1) I, \quad I \equiv \sum_{n=-\infty}^{+\infty}|k, n\rangle\langle k, n|,
\end{aligned}
$$

commutes with all operators of the Lie algebra $s u(1,1)$ and is therefore proportional to the identity operator $I$ of the space of an irreducible representation and is called the Casimir operator for $s u(1,1)$. The left-hand sides of (8) represents two different factorizations of the main part of the operator of the differential equations. These are, in principle, the Infeld-Hull factorizations [43] and in next Section we find the factors explicitly for the Ultraspherical polynomials.

Abstract equations of the form $(C-k(k-1) I)|k, n\rangle=0$ are equivalent to 
the eigenvalue equations in concrete realizations of the states $|k, n\rangle$ by functions. Using then (8) which are also equivalent to the eigenvalue equations one obtains different factorizations $K_{-} K_{+}$and $K_{+} K_{-}$of the main operator part (Hamilton operator) in these equations.

\section{Lowering and Raising Operations for the Ultraspherical Polynomials and Their Relatives in Connection with SU $(1,1)$-Symmetry}

In the treatment of the problem of algebra of lowering and raising operators for the Ultraspherical polynomials $P_{n}^{(\alpha, \alpha)}(u)$ and its relatives $G_{n}^{\alpha}(x)$ it is enough to possess one such, for example, for the polynomials and then one can make the transition to the orthonormalized functions. If one has, for example, the lowering operator then one can make the transition to the raising operator by transition to the adjoint operator. In both cases of the orthonormalized functions $P_{n}^{(\alpha, \alpha)}(u)$ and $g_{n}^{\alpha}(x)$ one knows the lowering operator for the corresponding polynomials $P_{n}^{(\alpha, \alpha)}(u)$ and $G_{n}^{\alpha}(x)$.

The lowering operator for the polynomials $P_{n}^{(\alpha, \alpha)}(u)$ under fixed parameter $\alpha$ is given in (3.14) and the raising operator is also known. Starting from the relation for lowering in (3.14) one may make in straight-forward way the transition to the normalized functions $P_{n}^{(\alpha, \alpha)}(u)$ via their definition in (3.10) and find a lowering relation which can be written in the form

$$
\begin{aligned}
& \sqrt{\frac{2 n+2 \alpha-1}{2 n+2 \alpha+1}}\left\{(n+\alpha+1) u+\frac{1}{2}\left(\left(1-u^{2}\right) \frac{\partial}{\partial u}+\frac{\partial}{\partial u}\left(1-u^{2}\right)\right)\right\} p_{n}^{(\alpha, \alpha)}(u) \\
& =\sqrt{n(n+2 \alpha)} p_{n-1}^{(\alpha, \alpha)}(u),
\end{aligned}
$$

where we write in braces a sum of Hermitean operator and an anti-Hermitean operator that makes the transition to the raising operator by adjunction easier $\left(\left(\frac{\partial}{\partial u}\right)^{\dagger}=-\frac{\partial}{\partial u}\right)$.

The first operator $(n+\alpha+1) u$ in braces is not independent on $n$ and therefore not a linear operator in usual sense because it depends on the index of the function $p_{n}^{(\alpha, \alpha)}(u)$ to which it is applied and we have to take this into account and come in rigorous way by inspection of the general relations for the Lie algebra $s u(1,1)$ given in last Section to the raising relation

$$
\begin{aligned}
& \sqrt{\frac{2 n+2 \alpha+3}{2 n+2 \alpha+1}}\left\{(n+\alpha) u-\frac{1}{2}\left(\left(1-u^{2}\right) \frac{\partial}{\partial u}+\frac{\partial}{\partial u}\left(1-u^{2}\right)\right)\right\} p_{n}^{(\alpha, \alpha)}(u) \\
& =\sqrt{(n+1)(n+2 \alpha+1)} p_{n+1}^{\alpha}(u) .
\end{aligned}
$$

By comparison of the right-hand sides of (9.1) and (9.2) with the right-hand sides in (8.6) one finds the connection of the parameter $\alpha$ in $p_{n}^{(\alpha, \alpha)}(u)$ to the parameter $k$ in the abstract relations to be 


$$
k=\alpha+\frac{1}{2}=v
$$

The parameter $\alpha+\frac{1}{2}$ corresponds to the upper index $v$ of the Gegenbauer polynomials $C_{n}^{v}(u)$ according to (3.1) and thus this parameter $v$ can be exactly identified with the parameter $k$ in formulae (8.6). At the end of this Section we will make a remark how one may obtain genuine operators which do not depend on $n$ but this is more a principal action than an action to apply in praxis.

We discuss now the orthonormalized functions $g_{n}^{\alpha}(x)$ in this respect. From (6.8) follows using the definition of the orthonormalized functions $g_{n}^{\alpha}(x)$ in (5.13) the lowering relation which we may write using self-adoint operators in braces in the following form

$$
\begin{aligned}
& K_{-} g_{n}^{\alpha}(x) \\
& =\sqrt{\frac{2 n+2 \alpha-1}{2 n+2 \alpha+1}}\left\{\frac{(n+\alpha+1) x}{\sqrt{1+x^{2}}}+\frac{1}{2}\left(\sqrt{1+x^{2}} \frac{\partial}{\partial x}+\frac{\partial}{\partial x} \sqrt{1+x^{2}}\right)\right\} g_{n}^{\alpha}(x) \\
& =\sqrt{n(n+2 \alpha)} g_{n-1}^{\alpha}(x) .
\end{aligned}
$$

Since the functions $g_{n}^{\alpha}(x)$ are orthonormalized the lowering relation (9.4) must possess the principal form given in (8.6) and this is only possible if we identify the parameter $k$ with $\alpha+\frac{1}{2}$ such as given in (9.3). The left-hand side of the operations has not yet a form independent on the number $n$ of the function $g_{n}^{\alpha}(x)$ but using the differential equations it should be possible to transform it into a form independent of $n$ that, however, we do not make here.

The operation of lowering in (9.4) was written in a form two sum terms where the first is a Hermitean (or self-adjoint) operator and the second an antiHermitean operator. By Hermitean conjugation we find from the left-hand side an operator which raise the index from $n-1$ to $n$ and if we rise the index $n$ by a step of 1 we find the following operation of raising

$$
\begin{aligned}
& K_{+} g_{n}^{\alpha}(x) \\
& =\sqrt{\frac{2 n+2 \alpha+3}{2 n+2 \alpha+1}}\left\{\frac{(n+\alpha) x}{\sqrt{1+x^{2}}}-\frac{1}{2}\left(\sqrt{1+x^{2}} \frac{\partial}{\partial x}+\frac{\partial}{\partial x} \sqrt{1+x^{2}}\right)\right\} g_{n}^{\alpha}(x) \\
& =\sqrt{(n+1)(n+2 \alpha+1)} g_{n+1}^{\alpha}(x) \text {. }
\end{aligned}
$$

The operations (9.1) and (9.4) and also (9.2) and (9.5) or widely analogous and may also be obtained by the coordinate transformation $u \leftrightarrow x$ given in (5.3). In Appendix $\mathrm{D}$ we compile these relations using non-Hermitean operators in braces and give the results for the calculations of $K_{-} K_{+}$and $K_{+} K_{-}$in the considered realizations of the operations $K_{-}$and $K_{+}$.

Many of the discussed relations are analogous to corresponding relations for 
Hermite polynomials although the last are much simpler ${ }^{6}$.

The lowering and raising operators $K_{-}$and $K_{+}$in the above relations were obtained in a form where they depend on the number $n$ of the eigenfunctions. Therefore, their application to an arbitrary function is only possible after decomposition of this function into the basis of the orthonormalized eigenfunctions. This can be removed by observing that the operator of the eigenvalue equation is quadratical in the numbers $n$ and is in abstract form of the kind

$$
\left\{\left(a n^{2}+2 b n+c\right) I-H\right\}|k, n\rangle=0,
$$

with coefficients $(a, b, c)$ and where $H$ is an operator which we call Hamilton operator. We may formally solve the quadratic Equation (9.6) for $n$ with the solution

$$
n \rightarrow \frac{1}{a}\left(-b I \pm \sqrt{\left(b^{2}-a c\right) I+a H}\right) .
$$

Inserting for $n$ the operator on the right-hand side in the formulae for the lowering and raising operator one obtains it in the general operator form. The sign has to be chosen in such a way that it corresponds to non-negative $n$. For example, in the realization of $s u(1,1)$ by the functions $p_{n}^{(\alpha, \alpha)}(u)$ which satisfy the second-order differential Equation (3.11) one has $a=1, b=2 \alpha+1, c=\alpha$ and the Hamilton operator corresponds in this realization to $H=\frac{(\alpha u)^{2}}{1-u^{2}}-\frac{\partial}{\partial u}\left(1-u^{2}\right) \frac{\partial}{\partial u}$. This shows that the lowering and raising operators for $s u(1,1)$ are, principally, well-defined linear operators but for practical purposes their application remains to be difficult.

${ }^{6}$ In case of the Hermite polynomials $H_{n}(x)$ the annihilation operator $a$ and creation operator $a^{\dagger}$ obey the commutation relation of a Heisenberg-Weyl algebra $w(2)$ and applied to abstract orthonormalized states $|n\rangle$ act by

$$
a|n\rangle=\sqrt{n}|n-1\rangle, \quad a^{\dagger}|n\rangle=\sqrt{n+1}|n\rangle, \quad a^{\dagger} a|n\rangle=n|n\rangle, \quad\langle m \mid n\rangle=\delta_{m, n}, \quad\left[a, a^{\dagger}\right]=I .
$$

The action of the annihilation and creation operators onto the orthonormalized Hermite functions $h_{n}(x)$ defined by

$$
h_{n}(x) \equiv \frac{1}{\pi^{\frac{1}{4}} \sqrt{2^{n} n !}} \exp \left(-\frac{x^{2}}{2}\right) H_{n}(x), \quad \int_{-\infty}^{+\infty} \mathrm{d} x h_{m}(x) h_{n}(x)=\delta_{m, n},
$$

as a realization of the abstract states $|n\rangle$ can be described by

$$
a: \frac{1}{\sqrt{2}}\left(x+\frac{\partial}{\partial x}\right) h_{n}(x)=\sqrt{n} h_{n-1}(x), \quad a^{\dagger}: \frac{1}{\sqrt{2}}\left(x-\frac{\partial}{\partial x}\right) h_{n}(x)=\sqrt{n+1} h_{n+1}(x),
$$

that means with operators on the left-hand side in a form independent of the index $n$ of the functions $h_{n}(x)$. This is connected with the differential equation for the Hermite functions $h_{n}(x)$ where its Hamilton operator $H$ can be factorized in different way

$$
\begin{aligned}
0 & =\left\{H-\left(n+\frac{1}{2}\right)\right\} h_{n}(x)=\left\{\frac{1}{2}\left(x^{2}-\frac{\partial^{2}}{\partial x^{2}}\right)-\left(n+\frac{1}{2}\right)\right\} h_{n}(x) \\
& =\left\{\frac{1}{2}\left(x-\frac{\partial}{\partial x}\right)\left(x+\frac{\partial}{\partial x}\right)-n\right\} h_{n}(x)=\left\{\frac{1}{2}\left(x+\frac{\partial}{\partial x}\right)\left(x-\frac{\partial}{\partial x}\right)-(n+1)\right\} h_{n}(x) .
\end{aligned}
$$




\section{Generating Functions for Jacobi Polynomials}

In this and the following Sections we derive some formulae for general Jacobi polynomials $P_{n}^{(\alpha, \beta)}(u)$. The best-known and basic linear generating function for general Jacobi polynomials is ([1] and, e.g., [3] [4] [5] [15])

$$
\begin{aligned}
& \sum_{n=0}^{\infty} t^{n} P_{n}^{(\alpha, \beta)}(u)=\frac{2^{\alpha+\beta}}{R\left((1-t+R)^{\alpha}(1+t+R)^{\beta}\right)}, \\
& R \equiv \sqrt{1-2 u t+t^{2}}, \quad(t<1) .
\end{aligned}
$$

According to citation by Szego [1] (p. 69) it goes back to Jacobi. In the monograph of Srivastava and Manocha [15] besides this linear generating function one may find also a bilinear generating function from Bailey (Equation (5) on p. 83) with a function $F_{4}$ defined on p. 66 (Equation 26). The problem with the generating function (1) is that for $\alpha=\beta$ it does not make the transition to the most simple basic generation function for the Ultraspherical polynomials $P_{n}^{(\alpha, \alpha)}(u)$ or Gegenbauer polynomials $C_{n}^{v}(u)$. Therefore, Rainville [10] (pp. 255, 256) and Andrews et al. [18] ((Equation 6.4.7) on p. 301 in [18]) (see also Koornwinder et al. [17], p. 449, Equation 18.12.3) derive yet another generating function for the Jacobi polynomials for $\sum_{n=0}^{\infty} \frac{(\alpha+\beta+1)_{n}}{(\beta+1)_{n}} t^{n} P_{n}^{(\alpha, \beta)}(u)$ with a complicated result expressed by the Hypergeometric function ${ }_{2} F_{1}(a, b ; c ; z)$ which, however, in case of $\alpha=\beta$ makes the transition to the following relatively simple basic generating function for Ultraspherical polynomials (Equation (6.4.8) in [18])

$$
\sum_{n=0}^{\infty} \frac{(n+2 \alpha) ! \alpha !}{(2 \alpha) !(n+\alpha) !} t^{n} P_{n}^{(\alpha, \alpha)}(u)=\frac{1}{\left(1-2 u t+t^{2}\right)^{\alpha+\frac{1}{2}}} .
$$

This generating function becomes particularly simple in terms of the Gegenbauer polynomials $C_{n}^{v}(u)$ according to their relation (3.1) to the Ultraspherical polynomials $P_{n}^{(\alpha, \alpha)}(u)$

$$
\sum_{n=0}^{\infty} t^{n} C_{n}^{v}(u)=\frac{1}{\left(1-2 u t+t^{2}\right)^{v}} .
$$

In most representations of Orthogonal polynomials this generating function is derived directly from a definition of the polynomials or it is used as basis for the definition of polynomials which generalize the Legendre polynomials and are called then Gegenbauer polynomials (e.g., [10]). This basic generating function alone justifies their independent introduction in comparison to the Ultraspherical polynomials as special case of the Jacobi polynomials with a separate name.

A further generating function for Jacobi polynomials which is remarkable for its symmetry and for its factorization into a product with factors depending only on one of the parameters $(\alpha, \beta)$ is (Koornwinder et al. [17], p. 449, Equation 18.12.2) 


$$
\sum_{n=0}^{\infty} \frac{t^{n}}{(n+\alpha) !(n+\beta) !} P_{n}^{(\alpha, \beta)}(u)=\frac{2^{\alpha} I_{\alpha}(\sqrt{2 t(u-1)})}{(\sqrt{2 t(u-1)})^{\alpha}} \frac{2^{\beta} I_{\beta}(\sqrt{2 t(u+1)})}{(\sqrt{2 t(u+1)})^{\beta}} .
$$

It can be easily derived using the explicit representation (2.2) of the Jacobi polynomials by reordering the arising double sum.

We direct now our attention to the following generating function for the Jacobi polynomials $P_{n}^{(\alpha-n, \beta-n)}(u)$ which can be derived from (2.7) as follows (see also [15], p. 82)

$$
\begin{aligned}
& \sum_{t=0}^{\infty} t^{n} P_{n}^{(\alpha-n, \beta-n)}(u) \\
& =\sum_{n=0}^{\infty}\left(\frac{t}{2}\right)^{n} \sum_{k=0}^{n} \frac{\alpha ! \beta !}{k !(n-k) !(\alpha-k) !(\beta-n+k) !}(u+1)^{k}(u-1)^{n-k} \\
& =\sum_{k=0}^{\infty} \frac{\alpha !}{k !(\alpha-k) !}\left(\frac{t(u+1)}{2}\right)^{k} \sum_{l=0}^{\infty} \frac{\beta !}{l !(\beta-l) !}\left(\frac{t(u-1)}{2}\right)^{l}
\end{aligned}
$$

where we made the substitution $n-k=l$ of the summation indices and made a reordering of the summation. Using the Taylor series for $(1+x)^{\gamma}$ one finds from (10.5)

$$
\sum_{n=0}^{\infty} t^{n} P_{n}^{(\alpha-n, \beta-n)}(u)=\left(1+\frac{t(u+1)}{2}\right)^{\alpha}\left(1+\frac{t(u-1)}{2}\right)^{\beta} .
$$

In contrast to (10.1), the upper indices of the Jacobi polynomials in this generating function are not fixed parameters.

From this one finds the following alternative formula for the polynomials $P_{n}^{(\alpha-n, \beta-n)}(u)$

$$
n ! P_{n}^{(\alpha-n, \beta-n)}(u)=\left\{\frac{\partial^{n}}{\partial t^{n}}\left(1+\frac{t(u+1)}{2}\right)^{\alpha}\left(1+\frac{t(u-1)}{2}\right)^{\beta}\right\}_{t=0} .
$$

In the special case $\alpha=\beta$ of ultraspherical polynomials we obtain from (10.6)

$$
\sum_{n=0}^{\infty} t^{n} P_{n}^{(\alpha-n, \alpha-n)}(u)=\left(1+t u+\frac{t^{2}\left(u^{2}-1\right)}{4}\right)^{\alpha}=\left(\left(1+\frac{t u}{2}\right)^{2}-\frac{t^{2}}{4}\right)^{\alpha} .
$$

Apart from bilinear and even trilinear generating functions for Jacobi polynomials we find in Chap. 2 of the monograph of Srivastava and Manocha [15] also more general generating functions from type (10.6) where the upper indices are not pure parameters. Furthermore, we mention that we did not find up to now a generating function for the general Jacobi polynomials which generalizes the generating function (7.16) for the Ultraspherical polynomials.

\section{Operator Ordering and Operator Disentanglement for General Jacobi Polynomials}

In some regions of physics, in particular, in quantum optics and in the theory of 
differential equations it is important to have for disposition some ordering relations of non-commuting operators. To illustrate this we give some simple and known examples. For the operators of differentiation $\frac{\partial}{\partial x}$ and of multiplication $x$ with the commutation relation

$$
\left[\frac{\partial}{\partial x}, x\right]=1
$$

which are characteristic for a Heisenberg-Weyl Lie algebra the normal ordering is particularly important. Normal ordering is if all functions of the multiplication operator $x$ are in front of powers of the differentiation operator $\frac{\partial}{\partial x}$ Such relations are, for example, the transition from anti-normal ordering to normal ordering for powers of both operators

$$
\frac{\partial^{n}}{\partial x^{n}} x^{m}=\sum_{j=0}^{\{m, n\}} \frac{m ! n !}{j !(m-j) !(n-j) !} x^{m-j} \frac{\partial^{n-j}}{\partial x^{n-j}},
$$

or the disentanglement of powers of the kind $\left(x \frac{\partial}{\partial x}\right)^{n}$ or $\left(\frac{\partial}{\partial x} x\right)^{n}$ according to

$$
\begin{aligned}
& \left(x \frac{\partial}{\partial x}\right)^{n}=\sum_{k=0}^{n} S(n, k) x^{k} \frac{\partial^{k}}{\partial x^{k}}, \\
& \left(\frac{\partial}{\partial x} x\right)^{n}=\frac{1}{x}\left(x \frac{\partial}{\partial x}\right)^{n} x=\sum_{k=0}^{n} S(n+1, k+1) x^{k} \frac{\partial^{k}}{\partial x^{k}},
\end{aligned}
$$

where $S(n, k)$ denotes the Stirling numbers of second kind which together with the Stirling numbers of first kind $s(n, k)$ obey the orthonormality relation (e.g., [45] [46])

$$
\sum_{k=0}^{\{m, n\}} S(m, k) s(k, n)=\delta_{m, n} .
$$

This relation makes it possible to find the conversion of the last operator identities. As a rule such operator identities can be proved by complete induction in connection with recurrence relations.

We now derive operator identities which arise specifically in connection with the theory of Jacobi polynomials. First we observe in connection with the weight function $w^{(\alpha, \beta)}(u)=(1-u)^{\alpha}(1+u)^{\beta}$ of the Jacobi polynomials the commutation relation

$$
\frac{\partial}{\partial u}(1-u)^{\alpha}(1+u)^{\beta}=(1-u)^{\alpha}(1+u)^{\beta}\left(\frac{\partial}{\partial u}+\frac{\alpha}{u-1}+\frac{\beta}{u+1}\right),
$$

and in generalization for powers of $\frac{\partial}{\partial u}$

$$
\frac{\partial^{n}}{\partial u^{n}}(1-u)^{\alpha}(1+u)^{\beta}=(1-u)^{\alpha}(1+u)^{\beta}\left(\frac{\partial}{\partial u}+\frac{\alpha}{u-1}+\frac{\beta}{u+1}\right)^{n} .
$$


Now we have the problem of disentanglement of the operator $\left(\frac{\partial}{\partial u}+\frac{\alpha}{u-1}+\frac{\beta}{u+1}\right)^{n}$ that means to bring it into a form where all powers of the differentiation operators $\frac{\partial}{\partial u}$ are to the right of functions $f(u)$ of the multiplication operator $u$. We begin with the solution of the partial problem for the operator $\left(\frac{\partial}{\partial u}+\frac{\alpha}{u-u_{0}}\right)^{n}$ where $\alpha$ and $u_{0}$ are arbitrary parameters. We conjecture from the explicit form of the disentanglement for low numbers $n$ the general relation

$$
\left(\frac{\partial}{\partial u}+\frac{\alpha}{u-u_{0}}\right)^{n}=\sum_{k=0}^{n} \frac{n ! \alpha !}{k !(n-k) !(\alpha-k) !} \frac{1}{\left(u-u_{0}\right)^{k}} \frac{\partial^{n-k}}{\partial u^{n-k}} .
$$

The correctness for arbitrary $n$ can be proved by complete induction and since this proof is relatively simple, we do not write it down here.

We mention that the operators $\frac{\partial}{\partial u}+\frac{\beta}{u+1}$ and the operator $\frac{\alpha}{u-1}$ possess the commutation relation

$$
\left[\frac{\partial}{\partial u}+\frac{\beta}{u+1}, \frac{\alpha}{u-1}\right]=-\frac{\alpha}{(u-1)^{2}},
$$

independent on the value of the parameter $\beta$ and therefore also for $\beta=0$ that is for the operator $\frac{\partial}{\partial u}$. This means that we can apply the theorem (7) with the substitutions $\frac{\partial}{\partial u} \rightarrow \frac{\partial}{\partial u}+\frac{\beta}{u+1}$ and $\alpha \rightarrow \beta, u_{0} \rightarrow-1$ and find

$$
\begin{aligned}
& \left(\frac{\partial}{\partial u}+\frac{\alpha}{u-1}+\frac{\beta}{u+1}\right)^{n} \\
& =\sum_{k=0}^{n} \frac{n ! \alpha !}{k !(n-k) !(\alpha-k) !} \frac{1}{(u-1)^{k}}\left(\frac{\partial}{\partial u}+\frac{\beta}{u+1}\right)^{n-k} \\
& =\sum_{k=0}^{n} \frac{n ! \alpha !}{k !(n-k) !(\alpha-k) !} \frac{1}{(u-1)^{k}} \sum_{l=0}^{n-k} \frac{(n-k) ! \beta !}{l !(n-k-l)(\beta-l) !} \frac{1}{(u+1)^{l}} \frac{\partial^{n-k-l}}{\partial u^{n-k-l}} \\
& =\sum_{j=0}^{n} \frac{n !}{(n-j) !}\left(\sum_{k=0}^{j} \frac{\alpha ! \beta !}{k !(j-k) !(\alpha-k) !(\beta-j+k) !} \frac{1}{(u-1)^{k}(u+1)^{j-k}}\right) \frac{\partial^{n-j}}{\partial u^{n-j}},
\end{aligned}
$$

where we made the substitution $j=k+l$ of the summation indices and applied a reordering of the sum terms in the finite sums. By comparison with (2.7) we find that the inner sum can be evaluated according to

$$
\begin{aligned}
& \sum_{k=0}^{j} \frac{\alpha ! \beta !}{k !(j-k) !(\alpha-k) !(\beta-j+k) !} \frac{1}{(u-1)^{k}(u+1)^{j-k}} \\
& =\left(\frac{2}{u^{2}-1}\right)^{j} P_{j}^{(\alpha-j, \beta-j)}(u),
\end{aligned}
$$

and the disentanglement relation (11.9) to "normal ordering" can be written in 
the following final form

$$
\frac{1}{n !}\left(\frac{\partial}{\partial u}+\frac{\alpha}{u-1}+\frac{\beta}{u+1}\right)^{n}=\sum_{j=0}^{n}\left(\frac{2}{u^{2}-1}\right)^{j} P_{j}^{(\alpha-j, \beta-j)}(u) \frac{1}{(n-j) !} \frac{\partial^{n-j}}{\partial u^{n-j}} .
$$

This is an operator identity which may be applied to arbitrary functions $f(u)$. Applying it to the function $f(u)=1$ we arrive at the definition (2.4) of the Jacobi polynomials $P_{n}^{(\alpha-n, \beta-n)}(u)$ after the substitutions

$(\alpha \rightarrow \alpha-n, \beta \rightarrow \beta-n)$ there.

Using the Taylor series of the exponential function we may derive from $(11.11)$

$$
\exp \left\{t\left(\frac{\partial}{\partial u}+\frac{\alpha}{u-1}+\frac{\beta}{u+1}\right)\right\}=\sum_{j=0}^{\infty}\left(\frac{2 t}{u^{2}-1}\right)^{j} P_{j}^{(\alpha-j, \beta-j)}(u) \exp \left(t \frac{\partial}{\partial u}\right),
$$

where we changed in an intermediate step the order of summations in the double sum. For the sum on the right-hand side of (11.11) we may apply the generating function (10.6) and we find the operator identity in the form ${ }^{7}$

$$
\begin{aligned}
& \exp \left\{t\left(\frac{\partial}{\partial u}+\frac{\alpha}{u-1}+\frac{\beta}{u+1}\right)\right\} \\
& =\left(1+\frac{t}{u-1}\right)^{\alpha}\left(1+\frac{t}{u+1}\right)^{\beta} \exp \left(t \frac{\partial}{\partial u}\right) \\
& =\exp \left\{\alpha \log \left(1+\frac{t}{u-1}\right)+\beta \log \left(1+\frac{t}{u+1}\right)\right\} \exp \left(t \frac{\partial}{\partial u}\right) .
\end{aligned}
$$

It is easy to see that this operator identity is correct in its Taylor series in powers of $t$ up to linear terms in $t$ and we checked by computer that this is also the case up to quadratic terms in $t$.

For an arbitrary function $\varphi(x)$ we find in similar way the more general operator identity

$$
\varphi\left\{t\left(\frac{\partial}{\partial u}+\frac{\alpha}{u-1}+\frac{\beta}{u+1}\right)\right\}=\sum_{j=0}^{\infty}\left(\frac{2 t}{u^{2}-1}\right)^{j} P_{j}^{(\alpha-j, \beta-j)}(u) \varphi^{(j)}\left(t \frac{\partial}{\partial u}\right)
$$

where $\varphi^{(j)}(x)$ the $j$-th derivative of $\varphi(x)$ which is to take for $x=t \frac{\partial}{\partial u}$ in the formula.

In the case $\alpha=\beta$ of Ultraspherical polynomials the operator disentanglement formulae (11.11) takes on the form

${ }^{7}$ This or (12) and formula (11) are analogous to corresponding operator identities for Hermite polynomials and thus for the Heisenberg-Weyl group as follows

$$
\frac{1}{n !}\left(\lambda^{2} 2 z-\frac{\partial}{\partial z}\right)^{n}=\sum_{k=0}^{n} \frac{\lambda^{n-k}}{(n-k) !} H_{n-k}(\lambda z) \frac{(-1)^{k}}{k !} \frac{\partial^{k}}{\partial z^{k}},
$$

with parameter $\lambda$ and due to $e^{A} e^{B}=e^{A+B+\frac{1}{2}[A, B]}$ for operators $A$ and $B$ if $[A,[A, B]]=[B,[A, B]]=0$

$$
\exp \left\{t\left(\lambda^{2} 2 z-\frac{\partial}{\partial z}\right)\right\}=\exp \left\{t \lambda^{2}(2 z-t)\right\} \exp \left(-t \frac{\partial}{\partial z}\right)
$$




$$
\frac{1}{n !}\left(\frac{\partial}{\partial u}+\frac{2 \alpha u}{u^{2}-1}\right)^{n}=\sum_{j=0}^{n}\left(\frac{2}{u^{2}-1}\right)^{j} P_{j}^{(\alpha-j, \alpha-j)}(u) \frac{1}{(n-j) !} \frac{\partial^{n-j}}{\partial u^{n-j}},
$$

and (11.13) simplifies to

$$
\exp \left\{t\left(\frac{\partial}{\partial u}+\frac{2 \alpha u}{u^{2}-1}\right)\right\}=\left(\frac{(u+t)^{2}-1}{u^{2}-1}\right)^{\alpha} \exp \left(t \frac{\partial}{\partial u}\right)
$$

In next section, we apply the derived operator identities to different functions and obtain in this way function identities.

\section{Derivation of Function Identities and a Convolution Identity for Jacobi Polynomials}

It was already said that the derived operator identities (11.11)-(11.16) can be applied to arbitrary functions $f(u)$ and one obtains then identities for functions represented on both sides of the equation in different way. In this way follows from (11.11)

$$
\frac{1}{n !}\left(\frac{\partial}{\partial u}+\frac{\alpha}{u-1}+\frac{\beta}{u+1}\right)^{n} f(u)=\sum_{j=0}^{n}\left(\frac{2}{u^{2}-1}\right)^{j} P_{j}^{(\alpha-j, \beta-j)}(u) \frac{f^{(n-j)}(u)}{(n-j) !} .
$$

For example, by applying (11.11) to the functions $\frac{u^{n}}{n !}$ one obtains the identity of the following functions on both side

$$
\begin{aligned}
& \frac{1}{n !}\left(\frac{\partial}{\partial u}+\frac{\alpha}{u-1}+\frac{\beta}{u+1}\right)^{n} u^{m} \\
& =\sum_{j=0}^{n}\left(\frac{2}{u^{2}-1}\right)^{j} P_{j}^{(\alpha-j, \beta-j)}(u) \frac{m ! u^{m-n+j}}{(n-j) !(m-n+j) !} .
\end{aligned}
$$

Since (12.1) and (12.2) are no more operator identities they cannot be further applied to functions written to the right on both sides. It is easy to check this identity in case of low integers $n$ (we made it up to $n=2$ ).

If we apply the operator identity (11.13) to an arbitrary function $f(u)$ we obtain

$$
\begin{aligned}
& \exp \left(t\left(\frac{\partial}{\partial u}+\frac{\alpha}{u-1}+\frac{\beta}{u+1}\right)\right) f(u) \\
& =\left(1+\frac{t}{u-1}\right)^{\alpha}\left(1+\frac{t}{u+1}\right)^{\beta} \exp \left(t \frac{\partial}{\partial u}\right) f(u) \\
& =\exp \left\{\alpha \log \left(1+\frac{t}{u-1}\right)+\beta \log \left(1+\frac{t}{u+1}\right)\right\} f(u+t) .
\end{aligned}
$$

By series expansion of both sides in powers of $t$ one can check this identity for low powers of $t$ (we made it up to powers $t^{2}$ ).

The main purpose of this Section is the application of the operator identity (11.11) to the function $\frac{1}{2^{n} n !}\left(u^{2}-1\right)^{n}$. Using then formula (2.3) which is in 
simple way equivalent to one of the basic definitions of the Jacobi polynomials one obtains the identity

$$
\begin{aligned}
P_{n}^{(\alpha, \beta)}(u) & =\left(\frac{\partial}{\partial u}+\frac{\alpha}{u-1}+\frac{\beta}{u+1}\right)^{n} \frac{1}{2^{n} n !}\left(u^{2}-1\right)^{n} \\
& =\sum_{j=0}^{n} \frac{n !}{(n-j) !}\left(\frac{2}{u^{2}-1}\right)^{j} P_{j}^{(\alpha-j, \beta-j)}(u) \frac{\partial^{n-j}}{\partial u^{n-j}} \frac{1}{2^{n} n !}\left(u^{2}-1\right)^{n} .
\end{aligned}
$$

If we now use the following definition of the Ultraspherical polynomials $P_{n-j}^{(j, j)}(u)$

$$
P_{n-j}^{(j, j)}(u)=\frac{1}{2^{n-j}(n-j) !} \frac{1}{\left(u^{2}-1\right)^{j}} \frac{\partial^{n-j}}{\partial u^{n-j}}\left(u^{2}-1\right)^{n},
$$

which follows directly from specialization of (3.3) and insert this into (12.4) we obtain the following identity of polynomials on the left-hand and on the righthand side.

$$
P_{n}^{(\alpha, \beta)}(u)=\sum_{j=0}^{n} P_{j}^{(\alpha-j, \beta-j)}(u) P_{n-j}^{(j, j)}(u) .
$$

This is a kind of convolution representation of arbitrary Jacobi polynomials $P_{n}^{(\alpha, \beta)}(u)$ and is similar to Vandermond's convolution identity for binomial coefficients.

Using the operator identity (11.11) with the substitutions $\alpha \rightarrow \alpha-\gamma$ and $\beta \rightarrow \beta-\delta$ and formula (2.6) which is equivalent to the definition of the Jacobi polynomials but contains two free parameters $\gamma$ and $\delta$ we may generalize the convolution identity (12.6) as follows

$$
P_{n}^{(\alpha, \beta)}(u)=\sum_{j=0}^{n} P_{j}^{(\alpha-\gamma-j, \beta-\delta-j)}(u) P_{n-j}^{(j+\gamma, j+\delta)}(u),
$$

with the two arbitrary parameters $\gamma$ and $\delta$. In particular, choosing $\gamma=\alpha$ and $\delta=\beta$ one obtains

$$
\begin{aligned}
& P_{n}^{(\alpha, \beta)}(u)=\sum_{j=0}^{n} P_{j}^{(-j,-j)}(u) P_{n-j}^{(j+\alpha, j+\beta)}(u), \\
& \left(P_{j}^{(-j,-j)}(u)=\delta_{j, 0}\right) .
\end{aligned}
$$

In connection with the derived function identities of the "convolution-type" we mention here yet the following identity of a similar type which, however, in simple way can be formulated only for the Gegenbauer polynomials $C_{n}^{v}(u)$ (Koornwinder et al. [17], p. 461, Equation 18.18.29)

$$
C_{n}^{v}(u)=\sum_{k=0}^{n} C_{k}^{\mu}(u) C_{n-k}^{v-\mu}(u)
$$

where $\mu$ is a parameter which can be chosen arbitrarily within wide limits. A simple derivation of this identity is possible starting from the generating function (3) in the following way 


$$
\begin{aligned}
\sum_{n=0}^{\infty} t^{n} C_{n}^{v}(u) & =\frac{1}{\left(1-2 u t+t^{2}\right)^{v}}=\frac{1}{\left(1-2 u t+t^{2}\right)^{\mu}} \frac{1}{\left(1-2 u t+t^{2}\right)^{v-\mu}} \\
& =\sum_{k=0}^{\infty} t^{k} C_{k}^{\mu}(u) \sum_{l=0}^{\infty} t^{l} C_{l}^{\nu-\mu}(u)=\sum_{n=0}^{\infty} t^{n} \sum_{k=0}^{n} C_{k}^{\mu}(u) C_{n-k}^{\nu-\mu}(u) .
\end{aligned}
$$

Relation (12.9) follows then by identification of the coefficients to equal powers $t^{n}$ of the arbitrary $t$ on both sides. In contrast to the derived convolution identities (12.7) the identities (12.9) do not involve the upper indices in the summation and it seems to be not easy to extend (12.9) to more general simple function identities for general Jacobi polynomials.

With the known values of the Ultraspherical polynomials for argument $u=0$ given in (4.8) the identity (12.6) takes on the form

$$
P_{n}^{(\alpha, \beta)}(0)=\sum_{k=0}^{n} \frac{(-1)^{k} n !}{2^{2 k} k !(n-k) !} P_{n-2 k}^{(\alpha-n+2 k, \beta-n+2 k)}(0) .
$$

In special case $\alpha=\beta$ this leads to the following sum evaluation

$$
\sum_{k=0}^{m} \frac{m !(2 m) ! \alpha !}{k !(m-k) !(2 m-k) !(\alpha-m+k) !}=\frac{(2 m+\alpha) !}{(m+\alpha) !},
$$

which is also possible with Vandermond's convolution identity. The special cases $u= \pm 1$ in (12.6) using (2.5) lead also to special cases of Vandermond's convolution identity. One may look to (12.6) as to a generalization of Vandermond's convolution identity for binomial coefficients and the reason for this becomes clear if we take into account that the polynomials $P_{j}^{(\alpha-j, \beta-j)}(u)$ are necessary for the representation of linear transformations in products of binomials.

\section{Conclusion}

We derived new functions and representations for Jacobi and for the Ultraspherical polynomials and of their special cases of Legendre polynomials and of Chebyshev polynomials of first and of second kind. A main aim was to find for the Jacobi polynomials an alternative definition in comparison to the Rodrigues definition but in analogy to the alternative definition of Hermite polynomials (see footnote in Section 5) where this alternative definition was very successful and led in the past also to a basic definition of the Laguerre 2D and Hermite 2D polynomials. This last has shown the way for a unification of Hermite and Laguerre polynomials on a common two-dimensional level with two-dimensional transformation relations between them and, interestingly, had essentially to use the Jacobi polynomials for their representation but not with fixed upper indices as parameters as mainly in present work. We could realize this programme only for the Ultraspherical polynomials as special case of the Jacobi polynomials. A key to a possible generalization of the approach presented in this article is to find a relation which generalizes in relation (6.1) the polynomials $G_{n}^{\alpha}(x)$ to more general polynomials which depend on parameters $\alpha$ and $\beta$ 
and which are generated from the monomials $x^{n}$ by an operator depending only the parameters $(\alpha, \beta)$ but not on the degree $n$ of the polynomials. From such a relation as (6.1) if it exists it is then necessary to make the transformation back to the Jacobi polynomials. Such alternative definitions possess the advantage that we may generate the polynomials of degree $n$ from the monomials $x^{n}$ by a common operator and many calculations can be separated then into corresponding calculations for the monomials $x^{n}$ and after this to the application of the common operator. The auxiliary set of polynomials $G_{n}^{\alpha}(x)$ and the recurrence and differentiation relation for them derived in this article are also interesting for their own.

A further aim was to establish explicitly the group-theoretical background of Ultraspherical polynomials in form of the $S U(1,1)$-group. This background was clear from the differential equations which besides the operator part including derivatives up to the second order contains an eigenvalue part which is quadratically dependent on the degree $n$ of the polynomials. In an earlier paper we proved in very general way that a Hamilton operator with quadratical energy spectrum $E_{n}$ in powers of $n$ has $S U(1,1)$ as background and in case of a linear spectrum this is the Heisenberg-Weyl group $W(2)$. The factual establishment of the relations of the algebra of lowering and raising operators of the Jacobi polynomials to $S U(1,1)$ can be made via the corresponding orthonormalized functions. Surely, this part can be generalized to the whole sets of Jacobi polynomials (e.g., general Pöschl-Teller potentials) but was explicitly made in present article only to its special case of Ultraspherical polynomials.

We established a new operator identity for the general case of the Jacobi polynomials which is a kind of operator disentanglement and insofar it is related to reordering of non-commuting operators to normal ordering (all differential operators behind the multiplication operators) and is important and well known in quantum optics for the annihilation and creation operators of the HeisenbergWeyl group and also in the theory of differential equations. Operator identities can be applied to arbitrary functions and they provide then function identities. In this way we could prove a kind of convolution theorem for the Jacobi polynomials with a certain similarity to the Vandermond convolution identity for binomial coefficients. Sometimes it was difficult to find out within the immense literature to polynomials whether or not a particular formula or approach is already known or is it novel and our main attention was directed to the correctness of the formulae.

The Jacobi polynomials $P_{n}^{(\alpha, \beta)}(u)$ play a main role in the formulae for the Weyl correspondence between classical phase-space functions to quantummechanical operators. This was shortly communicated in [41] and an article to this is in preparation.

\section{References}

[1] Szegö, G. (1959) Orthogonal Polynomials. 2nd Edition, American Mathematical Society, New York. (1st Ed., 1939.) 
[2] Jackson, D. (1941) Fourier Series and Orthogonal Polynomials. The Mathematical Association of America, Oberlin, Ohio. (Republished: Dover Publications, Mineola, N.Y. 2004.)

[3] Erdélyi, A. (1953) Higher Transcendental Functions. Vol. II, McGraw-Hill, New York.

[4] Magnus, W., Oberhettinger, F. and Soni, R.P. (1966) Formulas and Theorems for the Special Functions of Mathematical Physics. 3rd Edition, Springer, Berlin. https://doi.org/10.1007/978-3-662-11761-3

[5] Gradshteyn, I.S. and Ryzhik, I.M. (1963) Tables of Series, Products and Integrals. 4th Edition, Nauka, Moscow. (Many Translation into Other Languages and Later Editions.)

[6] Abramowitz, M. and Stegun, I. (1964) Handbook of Mathematical Functions, National Bureau of Standards. Dover Publication, New York, 1965.

[7] Kusnetsov, D.S. (1965) Spetsialnye funktsii (Special functions). 2nd Edition, Vysshaya Shkola, Moskva. (In Russian)

[8] Lebedev, N.N. (1963) Spetsialnye funktsii i ikh priloshenya. 2nd Edition, Fizmatgiz Moskva. (In Russian) English Translation under the Title: Special Functions and Their Applications, Prentice-Hall, New Jersey 1965, Republished: Dover, New York 1972.

[9] Nikiforov, A.F. and Uvarov, V.B. (1974) Osnovy teorii spezialnykh funkziy (Foundations of Special Functions). Nauka, Moskva. English Translation: Special Functions of Mathematical Physics, Birkhäuser, Boston, 1988.

[10] Rainville, E.D. (1960) Special Functions. Chelsea Publishing, Bronx.

[11] Luke, Y.L. (1969) The Special Functions and Their Approximations. Vol. I, Academic Press, New York.

[12] Chihara, Th. (1978) An Introduction to Orthogonal Polynomials. Gordon and Breach, New York. (Republished: Dover Publications, Mineola, N.Y., 2011.)

[13] Suyetin, P.K. (1988) Classical Orthogonal Polynomials. Nauka, Moskva. (In Russian)

[14] Suyetin, P.K. (1979) Orthogonal Polynomials in Two Variables. Nauka, Moskva. (In Russian)

[15] Srivastava, H.M. and Manocha, H.L. (1984) A Treatise on Generating Functions. Ellis Horwood, John Wiley, New York.

[16] Olver, F.W.J., Lozier, D.W., Boisvert, R.F. and Clark, Ch.W. (2010) NIST Handbook of Mathematical Functions. Cambridge University Press, Cambridge.

[17] Koornwinder, T.H., Wong, R., Koekoek, R. and Swarttouw, R.F. (2010) Orthogonal Polynomials. In: Olver, F.W.J., Lozier, D.W., Boisvert, R.F. and Clark, Ch.W., Eds. NIST Handbook of Mathematical Functions, Cambridge University Press, Cambridge.

[18] Andrews, G.E., Askey, R. and Roy, R. (1999) Special Functions. Cambridge University Press, Cambridge. https://doi.org/10.1017/CBO9781107325937

[19] Askey, R.A., Koornwinder, Th. and Schempp, W. (1984) Special Functions: Group Theoretical Aspects and Applications. D. Reidel (Kluwer Academic), Dordrecht. https://doi.org/10.1007/978-94-010-9787-1

[20] Carlson, B.C. (1977) Special Functions of Applied Mathematics. Academic Press, New York.

[21] Rivlin, Th. (1990) Chebyshev Polynomials. John Wiley, New York.

[22] Bell, W.W. (1968) Special Functions, Van Nostrand Reinhol, New York; Dover, 
Mineola (N.Y.) 2004.

[23] Wigner, E.P. (1959) Group Theory, Academic Press, New York. (First German Edition, 1931.)

[24] Weyl, H. (1931) Gruppentheorie und Quantenmechanik, (2. Auflage), Hirzel, Leipzig. (In English: The Theory of Groups and Quantum Mechanics, Dover, New York 1931.)

[25] Van der Waerden, B.L. (1932) Die gruppentheoretische Methode in der Quantenmechanik. Springer, Heidelberg. https://doi.org/10.1007/978-3-662-02187-3

[26] Vilenkin, N.J. (1968) Special Functions and the Theory of Group Representation. Providance, R.I.: American Mathematical Soc. (Russian Original: Nauka, Moskva, 1965.)

[27] Gel'fand, I.M., Minlos, R.A. and Shapiro, Z.Ya. (1958) Predstavleniya gruppy vrashtcheniy i gruppy Lorenza (Representations of the Rotation Group and of the Lorentz Group). Fizmatgiz, Moskva.

[28] Gel'fand, I.M. and Naimark, M.A. (1957) Unitarnyje predstavlenyia klassicheskikh grupp, (Unitary Representations of Classical Groups). Fizmatgiz, Moskva, 1950; German Translation, I.M. Gelfand, M.A. Neumark, Unitäre Darstellungen der Klassischen Gruppen, Akademie-Verlag, Berlin.

[29] Miller Jr., W. (1968) Lie Theory of Special Functions. Academic Press, New York.

[30] Miller Jr., W. (1975) Theory and Application of Special Functions. In: Askey, R.A., Ed., Academic Press, New York, 305-351.

[31] Miller Jr., W. (1977) Symmetry and Separation of Variables. Addison-Wesley, Reading.

[32] Van der Jeugt, J. (2002) 3nj-Coefficients and Orthogonal Polynomials of Hypergeometric Type. In: Koelink, E. and Van Assche, W., Eds., Orthogonal Polynomials and Special Functions, Springer, Leuven, 25-92.

[33] Koelink, E. and Van Assche, W. (2002) Orthogonal Polynomials and Special Functions. Springer, Leuven.

[34] Dunkl, Ch.F. and Xu, Y. (2001) Orthogonal Polynomials of Several Variables. Cambridge University Press, Cambridge. https://doi.org/10.1017/CBO9780511565717

[35] Wünsche, A. (2015) Quantum-Mechanical Cumulant Expansions and Their Application to Phase-Space and to Phase Distributions. Physica Scripta, 90, 074063. https://doi.org/10.1088/0031-8949/90/7/074063

[36] Erdélyi, A. (1953) Higher Transcendental Functions. Vol. I, McGraw-Hill, New York.

[37] Flügge, S. (1971) Practical Method in Quantum Mechanics. Springer, New York.

[38] Gol'dman, I.I. and Krivchenkov, V.D. (1957) Sbornik zadach po kvantovoy mekhanike. Moskva, Gostekhizdat. (In Russian) English Edition: Problems in Quantum Mechanics, Pergamon Press, Oxford, 1961; German Edition: Aufgabensammlung zur Quantenmechanik, Dt. Verl. d. Wiss., Berlin, 1963.

[39] Bateman, H. and Erdélyi, A. (1954) Tables of Integral Transforms. Vol. II, McGrawHill, New York.

[40] Wünsche, A. (2015) Generating Functions for Products of Special Laguerre 2D and Hermite 2D Polynomials. Applied Mathematics, 6, 2142-2168. https://doi.org/10.4236/am.2015.612188

[41] Wünsche, A. (2002) Operator Algebras to General One-Dimensional QuantumMechanical Potentials with Discrete Spectrum. Journal of Optics B: Quantum and Semiclassical Optics, 4, 359-379. https://doi.org/10.1088/1464-4266/4/6/301 
[42] Vourdas, A. and Wünsche, A. (1998) Resolutions of the Identity in Terms of Line Integrals of SU(1,1) Coherent States. Journal of Physics A: Mathematical and General, 31, 9341-9352. https://doi.org/10.1088/0305-4470/31/46/024

[43] Infeld, L. and Hull, T. (1951) The Factorization Method. Reviews of Modern Physics, 23, 21-68. https://doi.org/10.1103/RevModPhys.23.21

[44] Oldham, K.B. and Spanier, J. (1974) The Fractional Calculus. Academic Press, New York. (Republished: Dover Publications, Mineola, N.Y., 2006.)

[45] Riordan, J. (1958) An Introduction to Combinatorial Analysis. John Wiley, New York.

[46] van Lint, J.H. and Wilson, R.M. (1992) A Course in Combinatorics. Cambridge University Press, Cambridge. 
Appendix A. Transformation of the Explicit Sum Expression for the Jacobi Polynomials

Formula (2.1) can be transformed in the following way

$$
\begin{aligned}
& P_{n}^{(\alpha, \beta)}(u) \\
= & \sum_{k=0}^{n} \frac{(n+\alpha) !(n+\beta) !}{k !(n-k) !(n+\alpha-k) !(k+\beta) !}\left(\frac{u+1}{2}-1\right)^{n-k}\left(\frac{u+1}{2}\right)^{k} \\
= & \sum_{k=0}^{n} \frac{(n+\alpha) !(n+\beta) !}{k !(n-k) !(n+\alpha-k) !(k+\beta) !} \sum_{l=0}^{n-k} \frac{(-1)^{n-k-l}(n-k) !}{l !(n-k-l) !}\left(\frac{u+1}{2}\right)^{k+l} \\
= & (n+\beta) ! \sum_{j=0}^{n} \frac{(-1)^{n-j}}{j !(n-j) !}\left(\frac{u+1}{2}\right)^{j} \sum_{k=0}^{n} \frac{j !(n+\alpha) !}{k !(j-k) !(n+\alpha-k) !(k+\beta) !} \\
= & (-1)^{n}(n+\beta) ! \sum_{j=0}^{n} \frac{(-1)^{j}}{j !(n-j) !}\left(\frac{u+1}{2}\right)^{j} \frac{(n+\alpha+\beta+j) !}{(j+\beta) !(n+\alpha+\beta) !}
\end{aligned}
$$

which is identical with (2.8). In the last step we used the sum evaluation

$$
\sum_{k=0}^{\{\mu, v\}} \frac{\mu ! v !}{k !(\mu-k) !(v-k) !(k+\lambda) !}=\frac{(\mu+v+\lambda) !}{(\mu+\lambda) !(v+\lambda) !},
$$

which is the Vandermond convolution formula for the binomial coefficients

$$
\begin{aligned}
& \sum_{k=0}^{n}\left(\begin{array}{l}
n \\
k
\end{array}\right)\left(\begin{array}{l}
c-n \\
a-k
\end{array}\right) \\
& =\sum_{k=0}^{n} \frac{n !(c-n) !}{k !(n-k) !(a-k) !(c-n-a+k) !} \\
& =\frac{c !}{a !(c-a) !} \\
& =\left(\begin{array}{l}
c \\
a
\end{array}\right),
\end{aligned}
$$

in substituted variables.

\section{Appendix B. Evaluation of Two Finite Sums}

We prove in this Appendix by the same method the evaluation of two finite sums by expressions of multiplicative type.

First, we prove the correctness of the evaluation of the inner sum in (4.3) according to

$$
\begin{aligned}
& \sum_{k=0}^{2 j} \frac{(-2)^{k}(2 n+2 \alpha-k) !}{k !(n+\alpha-k) !(2 j-k) !} \\
& =\frac{(-1)^{j}(2 n+2 \alpha-2 j) !}{j !(n+\alpha-j) !} .
\end{aligned}
$$

One method for a proof is the following. We transform the sum as follows (the upper limit $2 j$ of the summation is here extended to $\infty$; intermediately we 
make the variable substitution $x=1+\varepsilon$ )

$$
\begin{aligned}
& \sum_{k=0}^{2 j} \frac{(-2)^{k}(2 n+2 \alpha-k) !}{k !(n+\alpha-k) !(2 j-k) !} \\
& =\lim _{x \rightarrow 1}\left\{\sum_{k=0}^{\infty} \frac{(-2)^{k}}{k !(n+\alpha-k) !} \frac{1}{\underbrace{x^{2 j-k}}_{\rightarrow 1}} \frac{\partial^{2 n+2 \alpha-2 j}}{\partial x^{2 n+2 \alpha-2 j}} x^{2 n+2 \alpha-k}\right\} \\
& =\lim _{x \rightarrow 1} \frac{\partial^{2 n+2 \alpha-2 j}}{\partial x^{2 n+2 \alpha-2 j}} x^{2 n+2 \alpha} \sum_{k=0}^{\infty} \frac{1}{k !(n+\alpha-k) !}\left(-\frac{2}{x}\right)^{k} \\
& =\frac{1}{(n+\alpha) !} \lim _{x \rightarrow 1} \frac{\partial^{2 n+2 \alpha-2 j}}{\partial x^{2 n+2 \alpha-2 j}} x^{2 n+2 \alpha}\left(\frac{x-2}{x}\right)^{n+\alpha} \mid(x=1+\varepsilon) \\
& =\frac{1}{(n+\alpha) !} \lim _{\varepsilon \rightarrow 0} \frac{\partial^{2 n+2 \alpha-2 j}}{\partial \varepsilon^{2 n+2 \alpha-2 j}} \underbrace{(-(1+\varepsilon)(1-\varepsilon))^{n+\alpha}}_{=\varepsilon^{2}-1} \\
& =\lim _{\varepsilon \rightarrow 0} \frac{\partial^{2(n+\alpha-j)}}{\partial \varepsilon^{2(n+\alpha-j)}} \sum_{l=0}^{\infty} \frac{(-1)^{l}}{l !(n+\alpha-l) !} \varepsilon^{2(n+\alpha-l)} \\
& =\frac{(-1)^{j}(2 n+2 \alpha-2 j) !}{j !(n+\alpha-j) !} .
\end{aligned}
$$

In the intermediate calculations we used fractional differentiation in case when the parameter $\alpha$ is not an integer. Fractional differentiation and integration is well founded (e.g., [39] [44]). A proof by complete induction from $j \rightarrow j+1$ which, however, is not simple seems also to be possible.

We found that the sum evaluation (B.1) is equivalent to the relations (4.8) and if we now calculate the values for $P_{n}^{(\alpha, \alpha)}(0)$ by specialization from the expansion (2.7) one finds that this is equivalent to the sum evaluations

$$
\begin{aligned}
& \sum_{k=0}^{2 j} \frac{(-1)^{k}(n+\alpha) !^{2}}{k !(2 j-k) !(n+\alpha-k) !(n+\alpha-2 j+k) !} \\
& =\frac{(-1)^{j}(n+\alpha) !}{j !(n+\alpha-j)}, \\
& \sum_{k=0}^{2 j+1} \frac{(-1)^{k}(n+\alpha) !^{2}}{k !(2 j+1-k) !(n+\alpha-k) !(n+\alpha-2 j-1+k) !}=0,
\end{aligned}
$$

which we have checked by computer but not independently proved.

Second, we prove the correctness of the evaluation of the inner sum in (5.1) according to

$$
\sum_{j=0}^{\left[\frac{n}{2}\right]} \frac{(-1)^{j}(2 n+2 \alpha-2 j) !}{(n-2 j) !(n+\alpha-j) !(j-k) !}=(-1)^{k} \frac{2^{n-2 k}(n+2 \alpha) !}{(n-2 k) !(k+\alpha) !},
$$

in analogous way as follows 


$$
\begin{aligned}
& \sum_{j=k}^{\left[\frac{n}{2}\right]} \frac{(-1)^{j}(2 n+2 \alpha-2 j) !}{(n-2 j) !(n+\alpha-j) !(j-k) !} \\
& =\lim _{x \rightarrow 1} \sum_{j=0}^{\infty} \frac{(-1)^{j}}{(j-k) !(n+\alpha-j) !} \frac{1}{x^{x^{n-2 j}}} \frac{\partial^{n+2 \alpha}}{\partial x^{n+2 \alpha}} x^{2 n+2 \alpha-2 j} \mid(j-k=l) \\
& =(-1)^{k} \lim _{x \rightarrow 1} \frac{\partial^{n+2 \alpha}}{\partial x^{n+2 \alpha}} x^{2 n+2 \alpha-2 k} \sum_{l=0}^{\infty} \frac{(-1)^{l}}{l !(n+\alpha-k-l) !} \frac{1}{x^{2 l}} \\
& =\frac{(-1)^{k}}{(n+\alpha-k) !} \lim _{x \rightarrow 1} \frac{\partial^{n+2 \alpha}}{\partial x^{n+2 \alpha}} x^{2(n+\alpha-k)}\left(\frac{x^{2}-1}{x^{2}}\right)^{n+\alpha-k} \mid(x=1+\varepsilon) \\
& =\frac{(-1)^{k}}{(n+\alpha-k) !} \lim _{\varepsilon \rightarrow 0} \frac{\partial^{n+2 \alpha}}{\partial \varepsilon^{n+2 \alpha}}(\varepsilon(2+\varepsilon))^{n+\alpha-k} \\
& =\frac{(-1)^{k}}{(n+\alpha-k) !} \lim _{\varepsilon \rightarrow 0} \frac{\partial^{n+2 \alpha}}{\partial \varepsilon^{n+2 \alpha}} \sum_{m=0}^{\infty} \frac{(n+\alpha-k) !}{m !(n+\alpha-k-m) !} 2^{m} \varepsilon^{2 n+2 \alpha-2 k-m} \\
& =(-1)^{k} \sum_{m=0}^{\infty} \frac{2^{m}(2 n+2 \alpha-2 k-m) !}{m !(n+\alpha-k-m) !(n-2 k-m) !} \lim _{\varepsilon \rightarrow 0} \varepsilon^{n-2 k-m} \\
& =(-1)^{k} \frac{2^{n-2 k}(n+2 \alpha) !}{(n-2 k) !(k+\alpha) !} .
\end{aligned}
$$

The calculated two sums are widely isolated and, apparently, cannot be extended to classes of sums with a continuous parameter, for example, substituting within the sum (1) $(-2)^{k} \rightarrow t^{k}$ in the sense that these sums may be evaluated also by a formula of multiplicative type.

Appendix C. Tables of Polynomials $G_{n}^{\alpha}(x)$ for $\alpha=-\frac{1}{2}, 0,+\frac{1}{2}$

We give here Tables for the initial members of the set of polynomials $G_{n}^{\alpha}(x)$ in the special cases $\alpha=-\frac{1}{2}, 0,+\frac{1}{2}$. The general relation to the Ultraspherical polynomials $P_{n}^{(\alpha, \alpha)}(u)$ is ((5.5) and (5.6))

$$
\begin{aligned}
G_{n}^{\alpha}(x) & =\frac{n ! \alpha !}{(n+\alpha) !}\left(\sqrt{1+x^{2}}\right)^{n} P_{n}^{(\alpha, \alpha)}\left(\frac{x}{\sqrt{1+x^{2}}}\right) \\
& =\frac{1}{2^{n}} \sum_{k=0}^{\left[\frac{n}{2}\right]} \frac{(-1)^{k} \alpha ! n !}{k !(k+\alpha) !(n-2 k) !}(2 x)^{n-k} \\
& =x^{n}{ }_{2} F_{1}\left(-\frac{n}{2},-\frac{n-1}{2} ; \alpha+1 ;-\frac{1}{x^{2}}\right),
\end{aligned}
$$

with the special cases $\alpha=-\frac{1}{2}, 0,+\frac{1}{2}$ and the relations to Chebyshev polynomials of first and second kind $T_{n}(u), U_{n}(u)$ and Legendre polynomials $P_{n}(u)$ according to 


$$
\begin{aligned}
G_{n}^{-\frac{1}{2}}(x) & =\left(\sqrt{1+x^{2}}\right)^{n} T_{n}\left(\frac{x}{\sqrt{1+x^{2}}}\right) \\
& =\frac{1}{2^{n}} \sum_{k=0}^{\left[\frac{n}{2}\right]} \frac{(-1)^{k}\left(-\frac{1}{2}\right) ! n !}{k !\left(k-\frac{1}{2}\right) !(n-2 k) !}(2 x)^{n-2 k}, \\
G_{n}^{0}(x) & =\left(\sqrt{1+x^{2}}\right)^{n} P_{n}\left(\frac{x}{\sqrt{1+x^{2}}}\right) \\
& =\frac{1}{2^{n}} \sum_{k=0}^{\left[\frac{n}{2}\right]} \frac{(-1)^{k} n !}{k !^{2}(n-2 k) !}(2 x)^{n-2 k}, \\
G_{n}^{\frac{1}{2}}(x) & =\frac{1}{n+1}\left(\sqrt{1+x^{2}}\right)^{n} U_{n}\left(\frac{x}{\sqrt{1+x^{2}}}\right) \\
& =\frac{1}{2^{n}} \sum_{k=0}^{\left[\frac{n}{2}\right]} \frac{(-1)^{k}\left(\frac{1}{2}\right) ! n !\left(k+\frac{1}{2}\right) !(n-2 k) !}{(2 x)^{n-2 k} .}
\end{aligned}
$$

Table of polynomials $G_{n}^{0}(x)$

$$
\begin{aligned}
& G_{0}^{0}(x)=1, \\
& G_{1}^{0}(x)=x, \\
& G_{2}^{0}(x)=x^{2}-\frac{1}{2}, \\
& G_{3}^{0}(x)=x^{3}-\frac{3}{2} x, \\
& G_{4}^{0}(x)=x^{4}-3 x^{2}+\frac{3}{8}, \\
& G_{5}^{0}(x)=x^{5}-5 x^{3}+\frac{15}{8} x, \\
& G_{6}^{0}(x)=x^{6}-\frac{15}{2} x^{4}+\frac{45}{8} x^{2}-\frac{5}{16}, \\
& G_{7}^{0}(x)=x^{7}-\frac{21}{2} x^{5}+\frac{105}{8} x^{3}-\frac{35}{16} x, \\
& G_{8}^{0}(x)=x^{8}-14 x^{6}+\frac{105}{4} x^{4}-\frac{35}{4} x^{2}+\frac{35}{128}, \\
& G_{9}^{0}(x)=x^{9}-18 x^{7}+\frac{189}{4} x^{5}-\frac{105}{4} x^{3}+\frac{315}{128} x .
\end{aligned}
$$

Tables of polynomials $G_{n}^{-\frac{1}{2}}(x)$ and $G_{n}^{\frac{1}{2}}(x)$ 


$$
\begin{aligned}
G_{0}^{-\frac{1}{2}}(x) & =1, & G_{0}^{\frac{1}{2}}(x) & =1 \\
G_{1}^{-\frac{1}{2}}(x) & =x, & G_{1}^{\frac{1}{2}}(x) & =x, \\
G_{2}^{-\frac{1}{2}}(x) & =x^{2}-1, & G_{2}^{\frac{1}{2}}(x) & =x^{2}-\frac{1}{3}, \\
G_{3}^{-\frac{1}{2}}(x) & =x^{3}-3 x, & G_{3}^{\frac{1}{2}}(x) & =x^{3}-x, \\
G_{4}^{-\frac{1}{2}}(x) & =x^{4}-6 x^{2}+1, & G_{4}^{\frac{1}{2}}(x) & =x^{4}-2 x^{2}+\frac{1}{5}, \\
G_{5}^{-\frac{1}{2}}(x) & =x^{5}-10 x^{3}+5 x, & G_{5}^{\frac{1}{2}}(x) & =x^{5}-\frac{10}{3} x^{3}+x, \\
G_{6}^{-\frac{1}{2}}(x) & =x^{6}-15 x^{4}+15 x^{2}-1, & G_{6}^{\frac{1}{2}}(x) & =x^{6}-5 x^{4}+3 x^{2}-\frac{1}{7}, \\
G_{7}^{-\frac{1}{2}}(x) & =x^{7}-21 x^{5}+35 x^{3}-7 x, & G_{7}^{\frac{1}{2}}(x) & =x^{7}-7 x^{5}+7 x^{3}-x, \\
G_{8}^{-\frac{1}{2}}(x) & =x^{8}-28 x^{6}+70 x^{4}-28 x^{2}+1, & G_{8}^{\frac{1}{2}}(x) & =x^{8}-\frac{28}{3} x^{6}+14 x^{4}-4 x^{2}+\frac{1}{9}, \\
G_{9}^{-\frac{1}{2}}(x) & =x^{9}-36 x^{7}+126 x^{5}-84 x^{3}+9 x . & G_{9}^{\frac{1}{2}}(x) & =x^{9}-12 x^{7}+\frac{126}{5} x^{5}-12 x^{3}+x .
\end{aligned}
$$

Appendix D. Compilation of $S U(1,1)$-Group Formulae for the Orthonormalized Systems of Functions $\boldsymbol{p}_{n}^{(\alpha, \alpha)}(\boldsymbol{u})$ and $\boldsymbol{g}_{n}^{\alpha}(\boldsymbol{x})$

We compile in this appendix the most important formulae for the algebra of lowering and raising of the indices $n$ of the two orthonormalized sytems of functions $p_{n}^{(\alpha, \alpha)}(u)$ and $g_{n}^{\alpha}(u)$ which are connected with the group $S U(1,1)$.

The basic definition of $p_{n}^{(\alpha, \alpha)}(u)$ is

$$
p_{n}^{(\alpha, \alpha)}(u)=\sqrt{\frac{2 n+2 \alpha+1}{2^{2 \alpha+1}} \frac{n !(n+2 \alpha) !}{(n+\alpha) !^{2}}}\left(1-u^{2}\right)^{\frac{\alpha}{2}} P_{n}^{(\alpha, \alpha)}(u) .
$$

The basic definition of the system of orthonormalized functions $g_{n}^{\alpha}(x)$ related with the functions $p_{n}^{(\alpha, \alpha)}(u)$ by variable transformation (5.3) is

$$
g_{n}^{\alpha}(x)=\sqrt{\frac{2 n+2 \alpha+1}{2^{2 \alpha+1}} \frac{(n+2 \alpha) !}{n ! \alpha !^{2}}}\left(1+x^{2}\right)^{-\left(\frac{n+\alpha}{2}+\frac{3}{4}\right)} G_{n}^{\alpha}(x),
$$

where $G_{n}^{\alpha}(x)$ is the set of polynomials

$$
G_{n}^{\alpha}(x) \equiv \sum_{k=0}^{\left[\frac{n}{2}\right]} \frac{(-1)^{k} \alpha ! n !}{k !(k+\alpha) !(n-2 k) !} \frac{x^{n-2 k}}{2^{2 k}} .
$$

The orthonormality relations are

$$
\int_{-1}^{+1} \mathrm{~d} u p_{m}^{(\alpha, \alpha)}(u) p_{n}^{(\alpha, \alpha)}(u)=\delta_{m, n}, \quad \int_{-\infty}^{+\infty} \mathrm{d} x g_{m}^{\alpha}(x) g_{n}^{\alpha}(x)=\delta_{m, n} .
$$

We will consider the functions $p_{n}^{(\alpha, \alpha)}(u)$. As lowering and raising operations according to 


$$
\begin{aligned}
K_{-} p_{n}^{(\alpha, \alpha)}(u) & \rightarrow \sqrt{n(n+2 \alpha)} p_{n-1}^{(\alpha, \alpha)}(u), \\
K_{+} p_{n}^{(\alpha, \alpha)}(u) & \rightarrow \sqrt{(n+1)(n+2 \alpha+1)} p_{n+1}^{(\alpha, \alpha)}(u),
\end{aligned}
$$

we derived the following realizations of $K_{-}$and $K_{+}{ }^{8}$

$$
\begin{aligned}
& K_{-}: \sqrt{\frac{2 n+2 \alpha-1}{2 n+2 \alpha+1}}\left\{(n+\alpha) u+\left(1-u^{2}\right) \frac{\partial}{\partial u}\right\} p_{n}^{(\alpha, \alpha)}(u) \\
& =\sqrt{n(n+2 \alpha)} p_{n-1}^{(\alpha, \alpha)}(u), \\
& K_{+}: \sqrt{\frac{2 n+2 \alpha+3}{2 n+2 \alpha+1}}\left\{(n+\alpha+1) u-\left(1-u^{2}\right) \frac{\partial}{\partial u}\right\} p_{n}^{(\alpha, \alpha)}(u) \\
& =\sqrt{(n+1)(n+2 \alpha+1)} p_{n+1}^{\alpha}(u) .
\end{aligned}
$$

According to

$$
\begin{aligned}
& K_{+} K_{-} p_{n}^{(\alpha, \alpha)}(u) \rightarrow n(n+2 \alpha) p_{n}^{(\alpha, \alpha)}(u), \\
& K_{-} K_{+} p_{n}^{(\alpha, \alpha)}(u) \rightarrow(n+1)(n+2 \alpha+1) p_{n}^{(\alpha, \alpha)}(u),
\end{aligned}
$$

from this follow the operations

$$
\begin{aligned}
& K_{+} K_{-}:\left\{(n+\alpha)\left((n+\alpha) u^{2}-\left(1-u^{2}\right)\right)-\left(\left(1-u^{2}\right) \frac{\partial}{\partial u}\right)^{2}\right\} p_{n}^{(\alpha, \alpha)}(u) \\
& =n(n+2 \alpha) p_{n}^{(\alpha, \alpha)}(u), \\
& K_{-} K_{+}:\left\{(n+\alpha+1)\left((n+\alpha+1) u^{2}+\left(1-u^{2}\right)\right)-\left(\left(1-u^{2}\right) \frac{\partial}{\partial u}\right)^{2}\right\} p_{n}^{(\alpha, \alpha)}(u) \\
& =(n+1)(n+2 \alpha+1) p_{n}^{(\alpha, \alpha)}(u) .
\end{aligned}
$$

Forming the difference $K_{-} K_{+}-K_{+} K_{-}=2 K_{0}$ which according to (8.6) is the commutator $\left[K_{-}, K_{+}\right]$equal to $2 K_{0}$ we obtain from (D.7) and (D.8) an obvious identity since according to (8.4) it has to give $2(n+k)=2 n+2 \alpha+1$ multiplied with the normalized function $p_{n}^{(\alpha, \alpha)}(u)$. Forming the Casimir operator $C=K_{0}^{2}-\frac{1}{2}\left(K_{-} K_{+}+K_{+} K_{-}\right)$and using that it has to be equal to $k(k-1)=\alpha^{2}-\frac{1}{4}$ multiplied with the identity operator we obtain the differential Equation (3.9) for $p_{n}^{(\alpha, \alpha)}(u)$ multiplied by the factor $1-u^{2}$.

We consider now the system of functions $g_{n}^{\alpha}(x)$. As lowering and raising operations according to

$$
\begin{aligned}
& K_{-} g_{n}^{\alpha}(x) \rightarrow \sqrt{n(n+2 \alpha)} g_{n-1}^{\alpha}(x), \\
& K_{+} g_{n}^{\alpha}(x) \rightarrow \sqrt{(n+1)(n+2 \alpha+1)} g_{n+1}^{\alpha}(x),
\end{aligned}
$$

${ }^{8}$ We prefer here a shorter representation instead of the more symmetrical representation in Section 9. 
we derived the following realizations of $K_{-}$and $K_{+}$

$$
\begin{aligned}
& K_{-}: \sqrt{\frac{2 n+2 \alpha-1}{2 n+2 \alpha+1}}\left\{\frac{\left(n+\alpha+\frac{3}{2}\right) x}{\sqrt{1+x^{2}}}+\sqrt{1+x^{2}} \frac{\partial}{\partial x}\right\} g_{n}^{\alpha}(x) \\
& =\sqrt{n(n+2 \alpha)} g_{n-1}^{\alpha}(x), \\
& K_{+}: \sqrt{\frac{2 n+2 \alpha+3}{2 n+2 \alpha+1}}\left\{\frac{\left(n+\alpha-\frac{1}{2}\right) x}{\sqrt{1+x^{2}}}-\sqrt{1+x^{2}} \frac{\partial}{\partial x}\right\} g_{n}^{\alpha}(x) \\
& =\sqrt{(n+1)(n+2 \alpha+1)} g_{n+1}^{\alpha}(x) .
\end{aligned}
$$

According to

$$
\begin{aligned}
& K_{+} K_{-} g_{n}^{\alpha}(u) \rightarrow n(n+2 \alpha) g_{n}^{\alpha}(u), \\
& K_{-} K_{+} g_{n}^{\alpha}(u) \rightarrow(n+1)(n+2 \alpha+1) g_{n}^{\alpha}(u),
\end{aligned}
$$

from this follow the operations

$$
\begin{aligned}
& K_{+} K_{-}:\left\{\frac{\left(n+\alpha+\frac{3}{2}\right)\left(\left(n+\alpha-\frac{3}{2}\right) x^{2}-1\right)}{1+x^{2}}-3 x \frac{\partial}{\partial x}-\left(\sqrt{1+x^{2}} \frac{\partial}{\partial x}\right)^{2}\right\} g_{n}^{\alpha}(u) \\
& =n(n+2 \alpha) g_{n}^{\alpha}(u), \\
& K_{-} K_{+}:\left\{\frac{\left(n+\alpha-\frac{1}{2}\right)\left(\left(n+\alpha+\frac{5}{2}\right) x^{2}+1\right)}{1+x^{2}}-3 x \frac{\partial}{\partial x}-\left(\sqrt{1+x^{2}} \frac{\partial}{\partial x}\right)^{2}\right\} g_{n}^{\alpha}(x) \\
& =(n+1)(n+2 \alpha+1) g_{n}^{\alpha}(x) .
\end{aligned}
$$

Forming the difference and taking into account that applied to $g_{n}^{\alpha}(x)$ it multiplies them with factor $2 n+2 \alpha+1$ one find as it is necessary an obvious identity. Forming the Casimir operator $C=K_{0}^{2}-\frac{1}{2}\left(K_{-} K_{+}+K_{+} K_{-}\right)$from it and taking into account that it multiplies $g_{n}^{\alpha}(x)$ by the factor $\alpha^{2}-\frac{1}{4}$ this provides the differential Equation (5.14) for $g_{n}^{\alpha}(x)$ divided by the factor $1+x^{2}$. 
Submit or recommend next manuscript to SCIRP and we will provide best service for you:

Accepting pre-submission inquiries through Email, Facebook, LinkedIn, Twitter, etc. A wide selection of journals (inclusive of 9 subjects, more than 200 journals)

Providing 24-hour high-quality service

User-friendly online submission system

Fair and swift peer-review system

Efficient typesetting and proofreading procedure

Display of the result of downloads and visits, as well as the number of cited articles Maximum dissemination of your research work

Submit your manuscript at: http://papersubmission.scirp.org/

Or contact apm@scirp.org 